\title{
Understanding the cost of capital of logistics service providers: an empirical investigation of multiple contingency variables
}

\author{
Kerstin Lampe $\cdot$ Erik Hofmann
}

Received: 2 April 2014/ Accepted: 21 October 2014/Published online: 11 November 2014

(C) The Author(s) 2014. This article is published with open access at Springerlink.com

\begin{abstract}
The article analyzes the influence of company-, industry- and market-related variables on the cost of capital of logistics service providers, as well as on their systematic risk. Financial information has become more and more important in strategic decision making (especially in the international context); in addition of being a measure of performance, the cost of capital is an important variable for logistics service providers in decisions about investing capital and developing the appropriate strategy. In total, financial data on over 700 logistics service providers for a period of 10 years were analyzed applying multiple regression analysis. Our results show that the logistics service industry is rather nonvolatile from an investor's viewpoint. Microeconomic variables significantly influence the cost of capital of logistics service providers, whereas systematic risk is influenced by macroeconomic variables. In both cases, significance is strongly dependent on the services offered and financial structure of the companies, although the headquarters location is irrelevant. Main implication of our study underlines the specific interdependencies of strategic decision making and cost of capital of logistics service providers. As recent research made only little efforts in linking the fields of business logistics and corporate finance, we follow a broad research approach to give a first comprehensive overview on this interdisciplinary topic.
\end{abstract}

Keywords Cost of capital - Systematic risk - Key financial figures $\cdot$ Logistics service providers $\cdot$ Strategic decisions $\cdot$ Value creation

K. Lampe $(\bowtie) \cdot$ E. Hofmann

Chair of Logistics Management, University of St. Gallen,

Dufourstrasse 40a, 9000 St. Gallen, Switzerland

e-mail: kerstin.lampe@unisg.ch

E. Hofmann

e-mail: erik.hofmann@unisg.ch

\section{Introduction}

While logistics has become more and more critical for the success of manufacturing or retail companies [1], the market for logistics from a provider's perspective is highly competitive. In order to compete, logistics service providers (LSP) have to understand their customers (shippers), general economic developments, and their main competitors. By being aware of their internal resources and capabilities, LSPs can then make several strategic decisions within the competitive environment in order to achieve business objectives such as profitability, organizational success, and growth [2].

A strategy and its affiliated investments should aim at achieving returns over the cost of resources or capital, respectively. The success of an LSP's strategic decisions is hence largely dependent on its capability to make a profit that exceeds its cost of capital $(\mathrm{CoC})$ [3]. CoC refers to the cost of a company's fund (both debt and equity). It may be seen as the required rate of return on capital from an investor's point of view (shareholders), in which the expected return on (invested) capital under a certain risk must be greater than the $\mathrm{CoC}$. Apergis et al. [4] observed that "one of the key decisions a firm has to reach is the fundamental determination of its cost of capital. This has substantial impact on both the composition of the firm's operations and its profitability." CoC supports company valuation and strategy formulation [5] and allows for an integrated consideration of yield expectations and risks.

With regard to the challenges of LSPs, the consideration of $\mathrm{CoC}$ becomes more and more important [6] as it offers valuable information when pursing appropriate strategies and investments. The $\mathrm{CoC}$ of LSPs' competitors is also of major relevance for strategic decisions that are, e.g., concerned with mergers and acquisitions (M\&A) or 
cooperation [7], as it allows for the assessment of potential takeover targets or network partners, respectively. For example, $\mathrm{CoC}$ can be gathered to determine the monetary performance of LSPs (and their competitors) in terms of the economic value added (EVA).

The analysis of the influence of company, industry, and market characteristics, especially on stock price and systematic risk in general, has a long tradition in research. These factors, among others, were analyzed by Daugherty et al. [8], Elyasiani et al. [9], Driesprong et al. [10], Huang et al. [11], Abugri [12], Rapach et al. [13], Cavaglia et al. [14], Sadorsky [15], Kavussanos and Marcoulis [16], Kaneko and Lee [17], Fama and French [18], Ross [19], and King [20]. At present, it is unknown whether the appropriate findings of previous studies are also valid for LSPs or whether specific patterns can be observed. Based on recent findings in financial logistics research, which indicate significant differences in the financial structure of LSP industries [21-26] and also a higher exposure of LSPs to (financial) risks than other industry companies [27], we expect significant differences for the analyses of the leverage of company, industry, and market characteristics on the $\mathrm{CoC}$ and the systematic risk of LSPs.

Despite the relevance of $\mathrm{CoC}$, recent research has made little effort in the financial analysis of LSPs. Initial approaches were followed by Hofmann and Lampe [21], who analyzed the financial structure of LSPs, and Liu and Lyons [22], who investigated the relationship between the financial performance and service capabilities of LSPs. Comparable analyses were made by Töyli et al. [23], Panayides [24], Panayides and So [25], and Ellinger et al. [26]. The analyses revealed that the performance (e.g., in terms of profitability) of LSPs is largely dependent on the industry in which an LSP operates and that appropriate LSPs also show a heterogeneous financial structure that is affected by both macroeconomic developments (e.g., oil price shocks), and microeconomic attributes (e.g., selffinancing power). However, $\mathrm{CoC}$ has not yet been specifically researched in the context of logistics. Thus, we consider the absence of appropriate studies to underpin our contribution on elaborating contingency variables concerning the CoC of LSPs.

Based on the practical importance of the CoC for LSPs and the identified research gap, this article aims to answer the following research question:

To what extent are the cost of capital and the systematic risk of LSPs dependent on company, industry, and market characteristics?

As recent research has shown, on the one hand, LSPs' financial structure is very heterogeneous, which justifies the analysis of company characteristics (microeconomic variables in terms of resource-based considerations). On the other hand, the profitability, but also the financial structure of LSPs, is largely dependent on the industry in which they operate, which is in turn embedded in an overall economic context. This highlights the importance of external characteristics (macroeconomic variables in terms of market-based considerations) when examining the CoC of LSPs.

In order to explore the CoC of LSPs, an appropriate operationalization of this "unit of analysis" is needed. A common conceptualization of $\mathrm{CoC}$ is the weighted average cost of capital (WACC). It represents "the average cost of each dollar of financing" [28]. Frequently, it is considered as a performance benchmark [29] and includes both the cost of debt and the cost of equity. Whereas the determination of the cost of debt is rather simple because interest rates in the financial market are easily obtained [30], the calculation of the cost of equity presents some challenges as "neither the rate of return nor the risk of a risky asset can normally be observed" [31, 32]. By referring to the systematic risk (beta, $\beta$ ), which describes the relation of stock price to market index volatility and the relation of the assumed market risk to an investment or financing measure, the cost of equity can be determined in general, and also for LSPs in particular.

As only little effort has been made in recent research, we follow a broad, overview approach. The investigation of a variety of influencing factors will provide a first comprehensive overview of the determinants of LSPs' CoC and systematic risk. For that purpose, financial data on over 700 LSPs for a period of 10 years are analyzed. An initial analysis of the stock price development of quoted LSPs offers implications for the development of hypotheses concerned with the influencing factors of LSPs' CoC and systematic risk. The influence of company, industry, and market characteristics is then investigated by conducting multiple linear regression analyses. From a managerial perspective, our results should explain how external developments, but also internal characteristics of an LSP, influence its $\mathrm{CoC}$ and systematic risk. The findings are intended to provide further insights for the strategic decision making of LSPs from a corporate finance perspective.

This paper is structured as follows. The following Section 2 reveals the background of the research on the $\mathrm{CoC}$ of LSPs and gives an overview on the literature concerned with determinants of stock price, systematic risk, and $\mathrm{CoC}$, from a general and logistical point of view. Based on this review, the hypotheses and the analytical model are derived (Section 3). Section 4 describes the methodology and data collection. Section 5 presents the results of the analysis, which are discussed in Section 6, in addition to the limitations of the research. Section 7 summarizes the results, discusses the managerial implications, and makes recommendations for future research. 

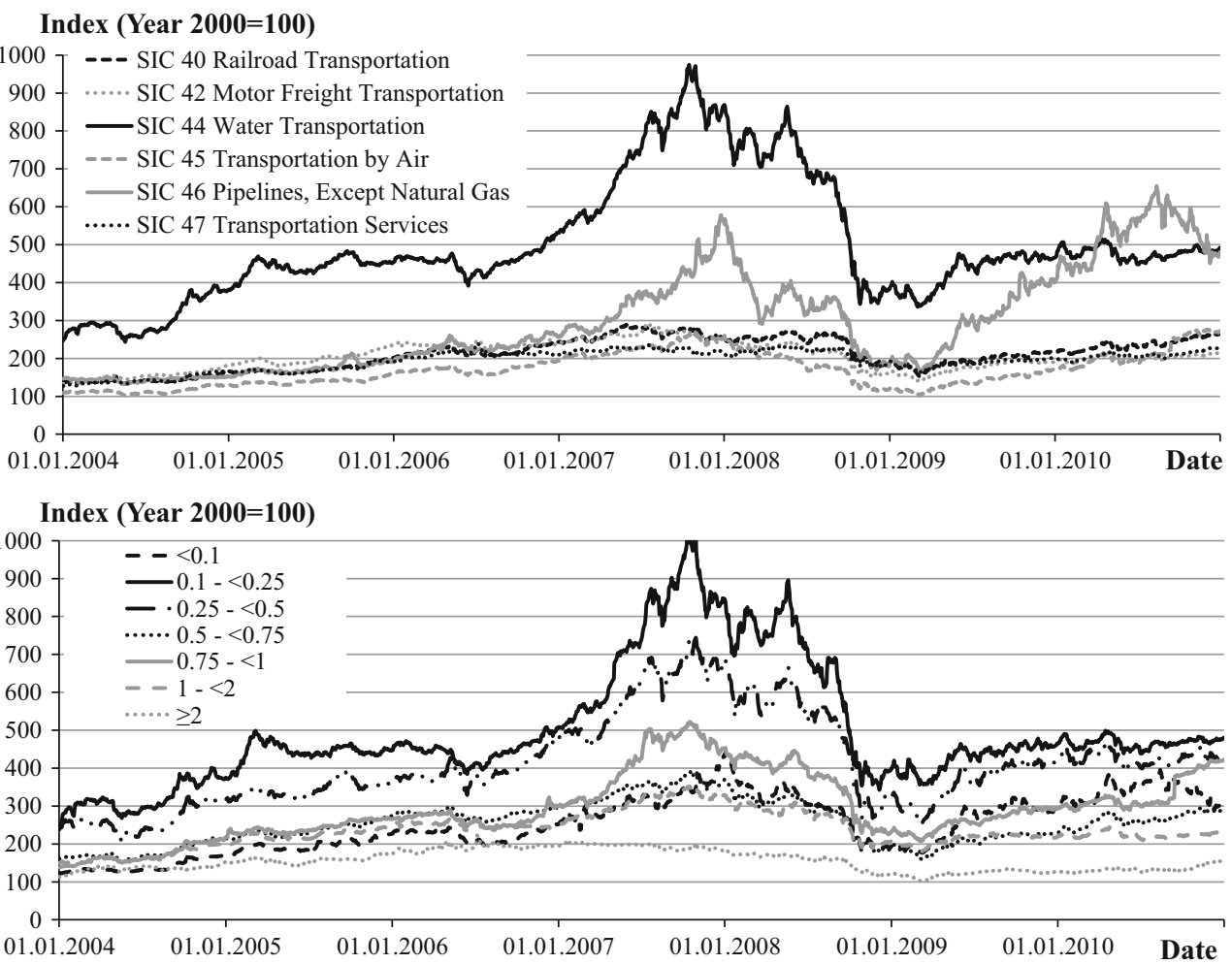

Fig. 1 Stock price performance of LSPs (2000-2010), clustered by a industry classification (SIC code) and $\mathbf{b}$ asset turnover (annual revenues to total assets). Only LSPs that have been continuously

\section{Background and literature review}

2.1 Why the cost of capital of logistics service providers matters

To date, research has not sufficiently considered LSPs from a corporate finance perspective. Relevant characteristics and determinants of $\mathrm{CoC}$ and systematic risk have been ignored:

- First, LSPs do not form a homogeneous group of companies, ${ }^{1}$ even when considering both their activity portfolios and their business risks. For example, aircraft carriers are more dependent on the oil price or global economic developments than railway companies.

- Second, LSPs show completely different financial structures [21]. For example, asset intensity (ratio of non-current assets to current assets) and the affiliated fiscal structure vary regarding both the general strategic orientation (e.g., "asset-light" vs. "asset-based"

\footnotetext{
${ }^{1}$ In the context of this work, a broad understanding of LSPs is taken; it includes carriers (basic services related to transportation using different modes of transport) as well as contract logistics providers offering bundled and customized services.
}

quoted since January 2000 have been included in the analysis (in total 503 LSPs). Data source is Thomson Datastream

approaches) and the form of corporate funding (e.g., debt- vs. equity-based funding).

To underscore our assumptions, we primarily analyze the stock price development of quoted LSPs, according to industry (on the Standard Industry Classification [SIC] basis), and company characteristics (e.g., asset turnover). Graphs (a) and (b) in Fig. 1 show obvious differences in the stock price developments of LSPs since the year 2000, according to cluster aggregation (e.g., SIC codes and level of asset turnover). All graphs have the same underlying values and differ only in categorizing the LSPs to different groups ("firm clusters"). The descriptive results of the analysis of the stock prices of LSPs indicate that the financial performance of an LSP depends on the industry sector in which the LSP operates as well as its capital structure. In contrast, the country in which the LSP's headquarters is located seems to be less important. ${ }^{2}$ These initial insights reaffirm our efforts to shed more light on the CoC of LSPs and its influencing contingency variables.

\footnotetext{
2 The analyses concerning the nationality (country in which the LSP's headquarters is located) are not presented in this study.
} 
2.2 Determinants of stock price, systematic risk, and cost of capital

Due to the close relationship between stock prices, systematic risk, and $\mathrm{CoC}$, which is primarily founded in their methods of calculation (Fig. 2), literature on these determinants is reviewed. Various general analyses have been conducted in the past.

a. Determinants of stock price: The influence of microeconomic variables related to firm size and equity ratios (company characteristics) on stock price was analyzed by Kavussanos and Marcoulis [16] and Fama and French [18], among others. The latter took a general financial perspective and analyzed the relationship between size and book-to-market equity (among other microeconomic variables), as well as stock returns (and the systematic risk), concluding that average stock returns are negatively related to systematic risk. In their analysis of industry characteristics on stock price returns from a general perspective, Isakov and Sonney [33] found that the industry in which a company operates has more explanatory power with regard to stock price returns than does the country where the company is located. They investigated ten industries, but not specifically logistics. Similar investigations were also made by Baca et al. [34] and Cavaglia et al. [14].

In a logistical context, Kavussanos and Marcoulis [16] investigated the stock market perception of different LSP industries (on the SIC basis) based on an analysis of microeconomic variables like the equity ratio. As a key result, they found that the influence of microeconomic variables on stock returns varies among the LSP industries. The influence of an LSP's industry (on the SIC basis) on its financial statement was analyzed by Hofmann and Lampe [21]. They revealed that the industry largely influences an LSP's financial structure, which is also closely linked to its stock price and CoC. The influence of macroeconomic variables (market characteristics) on stock price is considered in various disciplines, especially finance. The influence of macroeconomic variables (e.g., exchange rate, interest rate, money supply, industrial production, unemployment rate, and oil price) was addressed by Elyasiani et al. [9], who analyzed the influence of oil price shocks on different industry sectors (on the SIC basis), including the transportation industry. In most industries, they observed a significant relationship between oil-future return and industry returns. Comparable analyses were conducted by Driesprong et al. [10] and Sadorsky [15]. However, the results of previous analyses were not homogeneous. The results varied or were even contradictory, depending on the analyzed period and industry. Huang et al. [35], for example, identified a positive correlation between oil price development and stock returns for companies in the transportation sector, but not for S\&P 500 companies in general.

b. Determinants of systematic risk: The systematic risk represents "the percentage performance of the stock which has historically accompanied a one percent move in the market" [36]. Regarding the determinants of systematic risk, recent research has primarily focused on microeconomic variables (company characteristics). Iqbal and Shah [37] identified a negative correlation between liquidity, leverage, operating efficiency, dividend payout, market value of equity, and systematic risk and a positive correlation between profitability, firm size, growth, and systematic risk of companies from the non-financial sector. Hong and Sarkar [38] focused on the correlation between systematic risk and leverage ratio, earnings volatility, market price of risk, and growth options (positive correlation) as well as earnings growth rate, tax rate, and investments in expansion (negative correlation) in general, without differentiating between industries. Other analyses regarding both micro- and macroeconomic variables were conducted by Arfaoui and Abaoub [39] and Martikainen [40], also revealing the influence of both set of variables on systematic risk.

In a logistical context, Houmes et al. [41] analyzed the influence of the financial structure of trucking companies on their systematic risk and showed among other things, a positive influence of operating leverage on

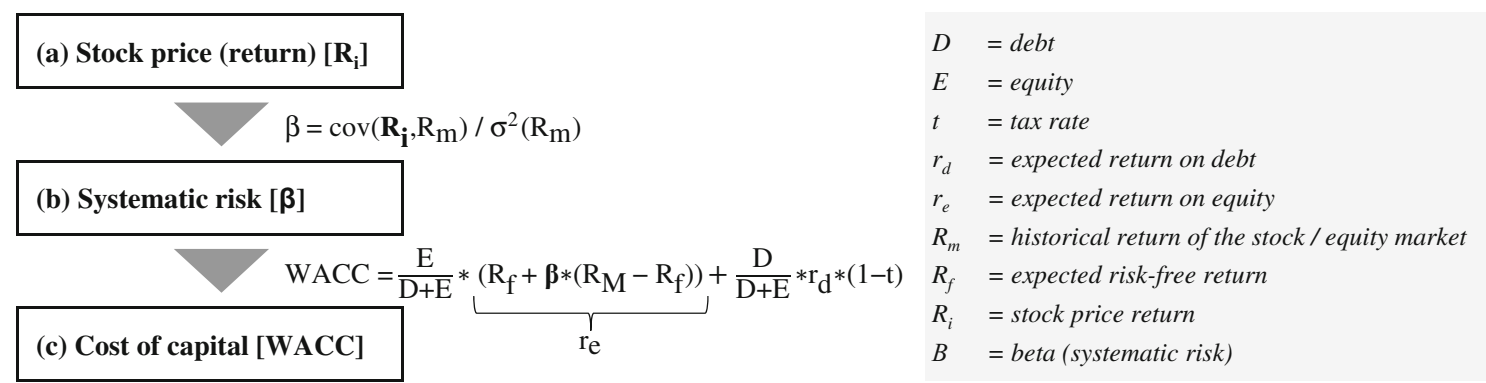

Fig. 2 Relationship between the determinants stock price, systematic risk, and cost of capital 
systematic risk. A similar analysis was conducted by MacArthur et al. [42]. Lu and Chen [43] proved that the systematic risk of LSPs is significantly dependent on the oil price risk, but varies between different industry sectors (e.g., water or air transportation). Based on their analysis of the influence of industry characteristics on stock price, Kavussanos and Marcoulis [44] investigated the systematic risk in the water transportation industry and found that its systematic risk is comparatively low. Allen et al. [45] showed that the deregulation of the US airline industry decreased as a result of deregulation (as a kind of market characteristic).

However, an analysis comparing the systematic risk of different LSP industries has not yet been conducted. Recent investigations have focused on the analysis of single LSP groups, but not their comparison. Such research would be of major interest to identify similarities and differences.

c. Determinants of cost of capital: Despite the close relationship between stock price, systematic risk, and $\mathrm{CoC}$, little recent research has analyzed the determinants of $\mathrm{CoC}$, especially in a logistical context. Bancel and Mittoo [46] analyzed the correlation between debt policy and $\mathrm{CoC}$ and found that it is influenced by the institutional environment and international operations. Sudarsanam [47] examined "the impact of the structural attributes of the industries on the cost of capital [...] within the capital asset pricing model" [47]. He showed that company characteristics like capital intensity (which was also considered on an aggregated level on an industry basis) significantly influence the systematic risk and the $\mathrm{CoC}$ of companies.

In general, the determinants-interpreted as contingency variables-can be distinguished by (1) company, (2) industry, and (3) market characteristics [14, $16,20]$. As our analysis shows, most research has considered the influence of industry, company, and market characteristics on stock price development and systematic risk, primarily on a general level. Despite the close link between systematic risk and $\mathrm{CoC}$, few investigations considering its determinants have been made in recent research. In logistics and transportation-oriented literature in particular, this aspect has been almost completely neglected.

\section{Development of hypotheses and theoretical model}

3.1 Hypotheses on the influence of contingency variables on the cost of capital

As the literature review has shown, specific analyses of factors influencing the $\mathrm{CoC}$ and systematic risk of LSPs have received little attention. Therefore, our research focuses on the impact of micro- and macroeconomic contingency variables on the $\mathrm{CoC}$ and systematic risk-as a key component of CoC-of LSPs.

Brooks and Del Negro [48], Isakov and Sonney [33], as well as Baca et al. [34] stated that country factors have lost importance and explanatory power, while industry factors are becoming more and more important in explaining the differences in financial performance. Referring first to findings in financial logistics research that revealed significant differences in the financial structure of LSP industries [21-26], we expect that the industry in which an LSP operates-especially the main mode of transport used-has a significant influence on its CoC. Such industry-specific impacts have been worked out by a variety of authors. For example, Gebhardt et al. [49] showed that "a firm's implied cost of capital is a function of its industry membership." Ghoul et al. [50] found that "[...] firms with better corporate social responsibility scores exhibit cheaper equity financing," whereas companies in the "sin industries" (tobacco and nuclear power) revealed reverse developments. Similarly, Rajan and Zingales [51] found that industrial sectors have different needs for external finance. Moreover, Fama and French [52] highlighted the influence of industry characteristics on the cost of equity. Finally, our introductory analysis of the stock prices of LSPs (Fig. 1a) indicates a strong influence of the "industry" in which a service provider operates (expressed by the predominant mode of transport used).

Based on this, hypothesis H1a states:

H1a The cost of capital of LSPs is significantly influenced by the predominant mode of transport.

Due to the close relationship between the $\mathrm{CoC}$ and the systematic risk of LSPs, we formulate hypothesis H1b as follows:

H1b The systematic risk of LSPs is significantly influenced by the predominant mode of transport.

It is obvious that microeconomic variables determine the total capital costs of a firm. The corporate discount rate (WACC) even depends largely on specific company characteristics (expressed by key financial figures of the firm), highlighted in a wide range of studies. For example, the analyses of Sudarsanam [47] showed a general influence of company characteristics on their CoC. Moreover, microeconomic variables are captured by various works on the "capital structure choice" [53]. In addition to the analysis of a firm's capital structure and the influence of microeconomic variables, Balakrishnan and Fox [54] showed a close relationship between $\mathrm{CoC}$ and a firm's strategy. 
Based on these coherences and our introductory analysis on the influence of microeconomic variables-the asset turnover - on stock prices of LSPs (Fig. 1b), we propose a second hypothesis $\mathrm{H} 2 \mathrm{a}$ as follows:

$\mathrm{H} 2 \mathrm{a}$ Microeconomic variables influence the cost of capital of LSPs.

We assume that the influencing microeconomic variables differ among the industries in which LSPs operates (=coherence to $\mathrm{H} 1 \mathrm{a}$ and $\mathrm{H} 1 \mathrm{~b}$ ). Moreover, the close relationship between systematic risk and $\mathrm{CoC}$ leads to the assumption that company characteristics also influence the systematic risk of LSPs, which was also shown in recent research results [37, 38].

$\mathrm{CoC}$ is calculated as the weighted sum of the cost of debt and cost of equity. Because the cost of debt and equity of LSPs are closely correlated with their financial structures, we suppose that microeconomic variables have a higher influence on $\mathrm{CoC}$ than on the systematic risk of LSPs. The minor influence of microeconomic variables on systematic risk was shown by Iqbal and Alisha [37], Rapack et al. [13], and Martikainen [40]. Based on this assumption, hypothesis $\mathrm{H} 2 \mathrm{~b}$ states:

$\mathrm{H} 2 \mathrm{~b}$ The influence of microeconomic variables on cost of capital is more significant than on systematic risk.

Several studies have shown the influence of market characteristics on the systematic risk of LSPs [39, 40, 42, 45], but a comparative analysis of all kinds of LSPs has not been conducted. Also investigating the differences between industry sectors, hypothesis $\mathrm{H} 3 \mathrm{a}$ states:

H3a Macroeconomic variables influence the systematic risk of LSPs.

Additionally, the close relationship between $\mathrm{CoC}$ and systematic risk leads to the assumption that market characteristics also influence the systematic risk of LSPs, which was also shown in recent research results [46].

Beta is calculated as the covariance of a company's stock price $\left(R_{\mathrm{i}}\right)$ and market index $\left(R_{\mathrm{m}}\right)$ divided by the variance of a market index [55]. Based on the direct influence of a market index, we suppose a higher influence of macroeconomic variables on the systematic risk than on the CoC of LSPs. Furthermore, Abugri [12] found that variables, such as exchange, interest rates, or money supply, significantly influence market returns, which are closely related to systematic risk. Similar results were presented by Chen et al. [56]. We therefore state hypothesis $\mathrm{H} 3 \mathrm{~b}$ as:

$\mathrm{H} 3 \mathrm{~b}$ The influence of macroeconomic variables on systematic risk is more significant than on cost of capital.

\subsection{Theoretical model of the analyses}

The consolidation of the hypotheses within a consistent model was done against the background of contingency theory, including contingency variables (context and response variables) that influence the performance of LSPs $[57,58]$. Contingencies represent the size, strategy, and environment of an LSP. As Grant [3] stated, analysis at the business strategy level can consider external influences (like market characteristics) as well as a company's resources (company characteristics). A resource-based consideration of the CoC of LSPs is conducted by analyzing the influence of microeconomic variables (company characteristics) on LSPs' performance in terms of CoC or systematic risk, while a market-based consideration acknowledges the dependency of a company's performance on its external (industry) environment. For this reason, the influence of macroeconomic variables (market characteristics) on LSPs' performance is also analyzed. Contingency theory allows for the integration of market- and resourcedbased considerations and structures the theoretical model for analysis (Fig. 3).

In addition to market and company characteristics, industry characteristics also influence the significance of determinants on stock price, $\mathrm{CoC}$, or systematic risk. For this reason, the differences between LSP industries (on the SIC basis) will be investigated in the model.

\section{Methodology}

\subsection{Approach and variables}

The methodology applied in this paper follows the work of Houmes et al. [41], Kavussanos and Marcoulis [16], Chen et al. [56]. and Fama and MacBeth [55], all of whom used very similar approaches. This research focuses on WACC - as a common conceptualization of $\mathrm{CoC}$ - and beta-as an indicator for systematic risk. The formulas for WACC [28] and beta [36] are presented in Fig. 2.

Data for WACC were directly adapted from the Thomson Datastream (a financial database that is accepted as valid and reliable); beta of the analyzed LSPs had to be calculated. Data on daily stock prices and market indices for a period of five years were used to calculate beta. For example, when calculating beta for the year 2010, daily data from 2006 to 2010 were applied. The S\&P 500 index is referred to as the market index. In this five-year span, the daily stock returns of each analyzed LSP are regressed on the corresponding returns for the S\&P 500.

To analyze the influence of micro- and macroeconomic variables on $\mathrm{CoC}$ and systematic risk, multiple linear 


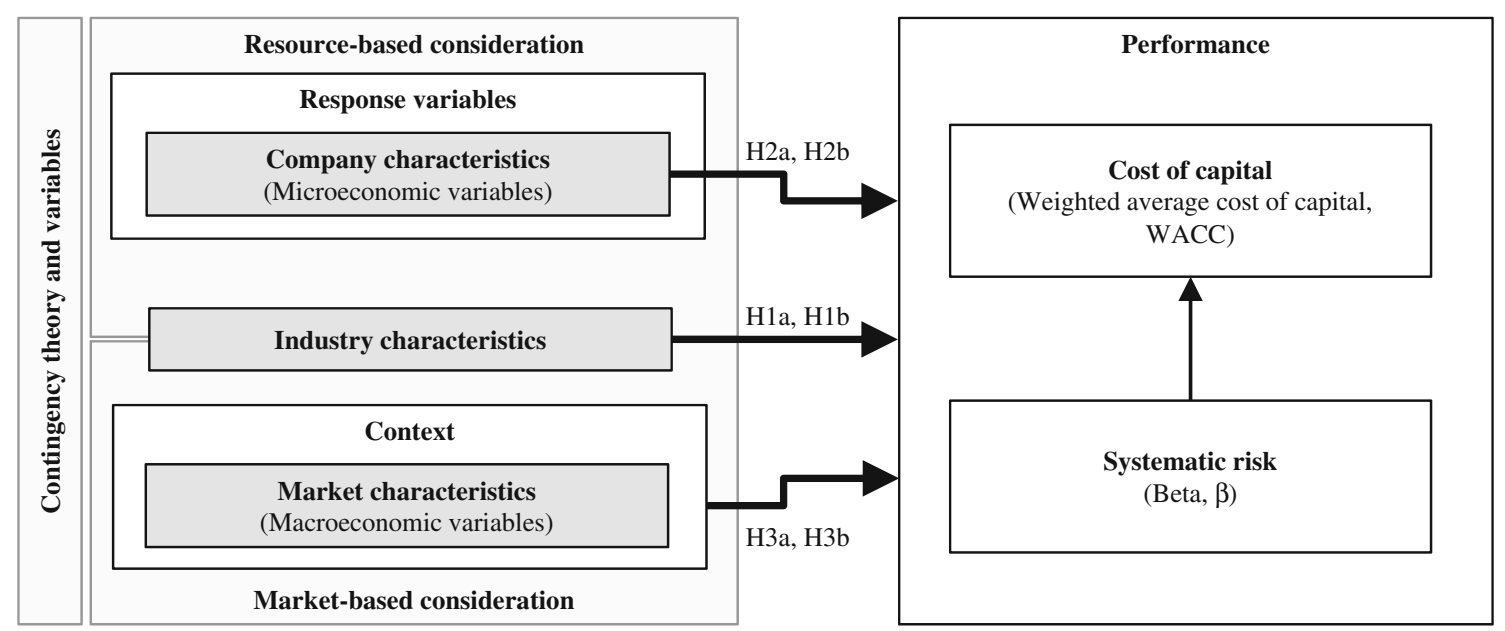

Fig. 3 Theoretical model used for the analysis of market, industry, and company characteristics on the performance of LSPs

regression analyses were conducted (for each hypothesis and corresponding clusters), where WACC or beta are the dependent variables and the micro- or macroeconomic variables are the independent variables. A stepwise regression was applied in order to identify the most significant variables influencing $\mathrm{CoC}$ and systematic risk [59]. In total, two sets of variables were used for the analyses. Detailed definitions or calculations of the chosen variables and related studies investigating the variables and their influence on stock prices, systematic risk, or $\mathrm{CoC}$, can be found in the Tables 11, 12 .

- Microeconomic variables: (a) related to asset structure: intensity of investment, asset intensity 1 , continuous intensity, asset intensity 2 , asset turnover, and current asset turnover; (b) related to capital structure: debt to equity ratio and equity ratio; (c) related to liquidity structure: current ratio and cash flow/sales; and (d) related to profitability structure: return on equity (ROE), return on assets (ROA), and net profit margin.

- Macroeconomic variables: labor force (total), gross capital formation (US\$), GNI (US\$), GDP (US\$), $\mathrm{CO}_{2}$ emissions (kilotons), employment to population ratio, adjusted net national income (US\$), money supply (money and quasi money M2, \% of GDP), and market capitalization (US\$) as well as the mean oil price (US\$).

The LSPs are analyzed as one group. In addition, the LSPs are classified according to industry sector in order to analyze the dependency on industry characteristics, and additionally, the geographical location of their headquarters. The analyses according to industry sectors are based on the Standard Industrial Classification (SIC). Analyses based on the SIC have found various applications in related logistics research $[9,16,44]$. Nevertheless, it has to be mentioned that the SIC only allows for an industry differentiation regarding the primarily used mode of transport or the offer of value-added services. A further differentiation in terms of carriers, third-party logistics (3PL), or fourth-party logistics (4PL) LSPs is not possible. LSPs with the following primary SIC codes were clustered and analyzed (by 2-place SIC code): SIC 40, Railroad Transportation; SIC 42, Motor Freight Transportation; SIC 44, Water Transportation (except SIC 448, Water Transportation of Passengers); SIC 45, Transportation by Air (except SIC 458, Airports, Flying Fields, and Airport Terminal); SIC 46, Pipeline except Natural Gas (this group includes LSPs that are concerned with pipeline transportation); and SIC 47, Transportation Services (except SIC 472, Arrangement of Passenger Transportation and SIC 474, Rental of Railroad Cars) [60]. The country clusters are based on the World Bank's country classification by income group [61]: high income/: non-OECD, high income: OECD, lower middle income, upper middle income.

Because of the availability of data related to our data set, the regression analyses of the WACC and macroeconomic variables refer to the period from 2006 to 2010; the regression analyses of systematic risk and macroeconomic variables refer to the period from 2000 to 2010. Macroeconomic data of the entire world and different country clusters were obtained from The World Bank's database (except oil price development). Oil price development is the mean of Brent Crude and WTI crude oil price development [62].

For conducting the regression analyses, the mean values of the WACC and beta of LSPs were used for each year, depending on the correspondent cluster group. The mean WACC and beta values of the SIC code clusters were analyzed against the values of macroeconomic variables that were valid for the whole world. The following regression equation was used: $Y=\alpha_{0}+\alpha_{1} X_{1}+\alpha_{2}$ $X_{2}+\cdots+\alpha_{\mathrm{j}} X_{\mathrm{j}}+e$, where $\alpha_{0}$ is the constant term, $\alpha_{\mathrm{j}}$ the 
regression coefficients, and $e$ an error term. In contrast to the usual terms of regression quotations, where $\beta$ defines the regression coefficient, in this case, $\alpha$ was chosen in order to avoid confusing systematic risk $(\beta)$ with the regression coefficient. In all tables depicting the results (Tables 1, 2, 3, 4, 5, 6), standardized regression coefficients are shown in order to enable the comparison of the coefficients in the same model.

\subsection{Sample characteristics}

We analyzed 702 LSPs from 70 countries all over the world. The LSPs have been chosen according to their primary SIC code and data availability. Because of data availability, some distinctions are made regarding each analysis:

- For the analysis of stock price quotations, only LSPs that were quoted since January 2000 (at least until December 2010) were included in the analysis: 503 LSPs.

- Data for the CoC (here WACC) of LSPs are used for the regression analyses of $\mathrm{CoC}$ and microeconomic variables. Values for the WACC and appropriate microeconomic variables were available for 437 out of the 702 LSPs. The characteristics (microeconomic

Table 1 Key financial figures (ratios) of logistics service providers used for regression analyses with WACC as the dependent variable

\begin{tabular}{|c|c|c|c|c|c|c|c|}
\hline \multicolumn{2}{|l|}{ Cluster description } & \multirow{2}{*}{$\begin{array}{l}\begin{array}{l}\text { Railroad } \\
\text { transportation }\end{array} \\
\text { SIC } 40\end{array}$} & \multirow{2}{*}{$\begin{array}{l}\text { Motor freight } \\
\text { transportation }\end{array}$} & \multirow{2}{*}{$\begin{array}{l}\text { Water } \\
\text { transportation }\end{array}$} & \multirow{2}{*}{$\begin{array}{l}\text { Transportation } \\
\text { by air }\end{array}$} & \multirow{2}{*}{$\begin{array}{l}\text { Pipeline, except } \\
\text { natural gas }\end{array}$} & \multirow{2}{*}{$\begin{array}{l}\begin{array}{l}\text { Transportation } \\
\text { services }\end{array} \\
\text { SIC } 47\end{array}$} \\
\hline $\begin{array}{l}\text { Cluster (SIC } \\
\text { code) }\end{array}$ & $\begin{array}{l}\text { All } \\
\text { LSPs }\end{array}$ & & & & & & \\
\hline Number of LSPs & 437 & 32 & 130 & 189 & 78 & 12 & 20 \\
\hline \multicolumn{8}{|c|}{ Ratios-outliers outside (3 standard deviations)| Mean value (standard deviation in parentheses) } \\
\hline Beta coefficient & $\begin{array}{l}0.31 \\
(0.50)\end{array}$ & $\begin{array}{l}0.50 \\
(0.55)\end{array}$ & $\begin{array}{l}0.16 \\
(0.47)\end{array}$ & $\begin{array}{l}0.33 \\
(0.51)\end{array}$ & $\begin{array}{l}0.33 \\
(0.41)\end{array}$ & $\begin{array}{l}0.43 \\
(0.23)\end{array}$ & $\begin{array}{l}0.58 \\
(0.50)\end{array}$ \\
\hline Cash flow/sales & $\begin{array}{l}17.67 \\
(15.45)\end{array}$ & $\begin{array}{l}19.97 \\
(10.60)\end{array}$ & $\begin{array}{l}11.07 \\
(10.21)\end{array}$ & $\begin{array}{l}24.37 \\
(17.79)\end{array}$ & $\begin{array}{l}12.88 \\
(10.01)\end{array}$ & $\begin{array}{l}28.32 \\
(18.58)\end{array}$ & $\begin{array}{l}5.19 \\
(4.26)\end{array}$ \\
\hline Current ratio & $\begin{array}{l}1.69 \\
(1.56)\end{array}$ & $\begin{array}{l}2.36 \\
(2.40)\end{array}$ & $\begin{array}{l}1.56 \\
(1.65)\end{array}$ & $\begin{array}{l}1.70 \\
(1.36)\end{array}$ & $\begin{array}{l}1.63 \\
(1.54)\end{array}$ & $\begin{array}{l}1.62 \\
(0.59)\end{array}$ & $\begin{array}{l}1.57 \\
(1.03)\end{array}$ \\
\hline $\begin{array}{l}\text { Intensity of } \\
\text { investment }\end{array}$ & $\begin{array}{l}0.53 \\
(0.25)\end{array}$ & $\begin{array}{l}0.69 \\
(0.20)\end{array}$ & $\begin{array}{l}0.49 \\
(0.24)\end{array}$ & $\begin{array}{l}0.59 \\
(0.24)\end{array}$ & $\begin{array}{l}0.47 \\
(0.24)\end{array}$ & $\begin{array}{l}0.59 \\
(0.24)\end{array}$ & $\begin{array}{l}0.21 \\
(0.19)\end{array}$ \\
\hline $\begin{array}{l}\text { Asset intensity } \\
1\end{array}$ & $\begin{array}{l}3.33 \\
(4.39)\end{array}$ & $\begin{array}{l}6.23 \\
(4.96)\end{array}$ & $\begin{array}{l}2.63 \\
(3.67)\end{array}$ & $\begin{array}{l}4.12 \\
(5.04)\end{array}$ & $\begin{array}{l}1.78 \\
(1.49)\end{array}$ & $\begin{array}{l}5.54 \\
(6.52)\end{array}$ & $\begin{array}{l}0.59 \\
(0.97)\end{array}$ \\
\hline $\begin{array}{l}\text { Continuous } \\
\text { intensity }\end{array}$ & $\begin{array}{l}0.32 \\
(0.20)\end{array}$ & $\begin{array}{l}0.20 \\
(0.18)\end{array}$ & $\begin{array}{l}0.33 \\
(0.18)\end{array}$ & $\begin{array}{l}0.28 \\
(0.18)\end{array}$ & $\begin{array}{l}0.38 \\
(0.21)\end{array}$ & $\begin{array}{l}0.25 \\
(0.21)\end{array}$ & $\begin{array}{l}0.58 \\
(0.21)\end{array}$ \\
\hline Asset intensity 2 & $\begin{array}{l}1.48 \\
(3.10)\end{array}$ & $\begin{array}{l}0.56 \\
(1.20)\end{array}$ & $\begin{array}{l}1.45 \\
(2.98)\end{array}$ & $\begin{array}{l}0.93 \\
(1.88)\end{array}$ & $\begin{array}{l}2.37 \\
(4.72)\end{array}$ & $\begin{array}{l}0.59 \\
(0.76)\end{array}$ & $\begin{array}{l}5.54 \\
(4.28)\end{array}$ \\
\hline Asset turnover & $\begin{array}{l}0.86 \\
(0.69)\end{array}$ & $\begin{array}{l}0.61 \\
(0.35)\end{array}$ & $\begin{array}{l}1.10 \\
(0.73)\end{array}$ & $\begin{array}{l}0.59 \\
(0.53)\end{array}$ & $\begin{array}{l}1.09 \\
(0.67)\end{array}$ & $\begin{array}{l}0.59 \\
(0.49)\end{array}$ & $\begin{array}{l}1.81 \\
(0.69)\end{array}$ \\
\hline $\begin{array}{l}\text { Current asset } \\
\text { turnover }\end{array}$ & $\begin{array}{l}3.00 \\
(1.68)\end{array}$ & $\begin{array}{l}3.48 \\
(1.35)\end{array}$ & $\begin{array}{l}3.49 \\
(1.67)\end{array}$ & $\begin{array}{l}2.30 \\
(1.45)\end{array}$ & $\begin{array}{l}3.38 \\
(1.56)\end{array}$ & $\begin{array}{l}3.21 \\
(2.42)\end{array}$ & $\begin{array}{l}4.14 \\
(1.67)\end{array}$ \\
\hline $\begin{array}{l}\text { Debt to equity } \\
\text { ratio }\end{array}$ & $\begin{array}{l}0.91 \\
(0.99)\end{array}$ & $\begin{array}{l}1.18 \\
(1.04)\end{array}$ & $\begin{array}{l}0.75 \\
(0.76)\end{array}$ & $\begin{array}{l}0.95 \\
(1.03)\end{array}$ & $\begin{array}{l}1.13 \\
(1.20)\end{array}$ & $\begin{array}{l}0.91 \\
(0.76)\end{array}$ & $\begin{array}{l}0.32 \\
(0.56)\end{array}$ \\
\hline Equity ratio & $\begin{array}{l}0.67 \\
(0.24)\end{array}$ & $\begin{array}{l}0.57 \\
(0.20)\end{array}$ & $\begin{array}{l}0.73 \\
(0.19)\end{array}$ & $\begin{array}{l}0.64 \\
(0.24)\end{array}$ & $\begin{array}{l}0.63 \\
(0.26)\end{array}$ & $\begin{array}{l}0.66 \\
(0.22)\end{array}$ & $\begin{array}{l}0.84 \\
(0.25)\end{array}$ \\
\hline ROE & $\begin{array}{l}0.11 \\
(0.09)\end{array}$ & $\begin{array}{l}0.12 \\
(0.09)\end{array}$ & $\begin{array}{l}0.09 \\
(0.08)\end{array}$ & $\begin{array}{l}0.11 \\
(0.09)\end{array}$ & $\begin{array}{l}0.15 \\
(0.10)\end{array}$ & $\begin{array}{l}0.13 \\
(0.10)\end{array}$ & $\begin{array}{l}0.11 \\
(0.10)\end{array}$ \\
\hline ROA & $\begin{array}{l}0.05 \\
(0.04)\end{array}$ & $\begin{array}{l}0.05 \\
(0.04)\end{array}$ & $\begin{array}{l}0.04 \\
(0.03)\end{array}$ & $\begin{array}{l}0.05 \\
(0.04)\end{array}$ & $\begin{array}{l}0.05 \\
(0.04)\end{array}$ & $\begin{array}{l}0.05 \\
(0.02)\end{array}$ & $\begin{array}{l}0.05 \\
(0.05)\end{array}$ \\
\hline $\begin{array}{l}\text { Net profit } \\
\text { margin }\end{array}$ & $\begin{array}{l}0.10 \\
(0.13)\end{array}$ & $\begin{array}{l}0.10 \\
(0.08)\end{array}$ & $\begin{array}{l}0.07 \\
(0.10)\end{array}$ & $\begin{array}{l}0.15 \\
(0.16)\end{array}$ & $\begin{array}{l}0.07 \\
(0.09)\end{array}$ & $\begin{array}{l}0.13 \\
(0.09)\end{array}$ & $\begin{array}{l}0.03 \\
(0.03)\end{array}$ \\
\hline WACC & $\begin{array}{l}0.0838 \\
(0.0783)\end{array}$ & $\begin{array}{l}0.0814 \\
(0.0568)\end{array}$ & $\begin{array}{l}0.0703 \\
(0.0632)\end{array}$ & $\begin{array}{l}0.0832 \\
(0.0842)\end{array}$ & $\begin{array}{l}0.1033 \\
(0.0764)\end{array}$ & $\begin{array}{l}0.0746 \\
(0.0715)\end{array}$ & $\begin{array}{l}0.1125 \\
(0.1202)\end{array}$ \\
\hline
\end{tabular}


Table 2 Key financial figures (ratios) of logistics service providers used for regression analyses with beta as the dependent variable

\begin{tabular}{|c|c|c|c|c|c|c|c|}
\hline \multicolumn{2}{|l|}{ Cluster description } & \multirow{2}{*}{$\begin{array}{l}\begin{array}{l}\text { Railroad } \\
\text { transportation }\end{array} \\
\text { SIC } 40\end{array}$} & \multirow{2}{*}{$\begin{array}{l}\begin{array}{l}\text { Motor freight } \\
\text { transportation }\end{array} \\
\text { SIC } 42\end{array}$} & \multirow{2}{*}{$\begin{array}{l}\begin{array}{l}\text { Water } \\
\text { transportation }\end{array} \\
\text { SIC } 44\end{array}$} & \multirow{2}{*}{$\begin{array}{l}\text { Transportation } \\
\text { by air }\end{array}$} & \multirow{2}{*}{$\begin{array}{l}\text { Pipeline, except } \\
\text { natural gas }\end{array}$} & \multirow{2}{*}{$\begin{array}{l}\begin{array}{l}\text { Transportation } \\
\text { services }\end{array} \\
\text { SIC } 47\end{array}$} \\
\hline Cluster (SIC code) & $\begin{array}{l}\text { All } \\
\text { LSPs }\end{array}$ & & & & & & \\
\hline Number of LSPs & 702 & 48 & 187 & 337 & 140 & 23 & 25 \\
\hline \multicolumn{8}{|c|}{ Ratios-outliers outside (3 standard deviations) I Mean value (standard deviation in parentheses) } \\
\hline Beta coefficient & $\begin{array}{l}0.32 \\
(0.63)\end{array}$ & $\begin{array}{l}0.38 \\
(0.50)\end{array}$ & $\begin{array}{l}0.18 \\
(0.45)\end{array}$ & $\begin{array}{l}0.33 \\
(0.73)\end{array}$ & $\begin{array}{l}0.41 \\
(0.61)\end{array}$ & $\begin{array}{l}0.38 \\
(0.28)\end{array}$ & $\begin{array}{l}0.52 \\
(0.48)\end{array}$ \\
\hline Cash flow/sales & $\begin{array}{l}13.99 \\
(35.02)\end{array}$ & $\begin{array}{l}19.47 \\
(14.36)\end{array}$ & $\begin{array}{l}8.84 \\
(23.05)\end{array}$ & $\begin{array}{l}18.66 \\
(43.00)\end{array}$ & $\begin{array}{l}9.86 \\
(14.31)\end{array}$ & $\begin{array}{l}24.66 \\
(23.85)\end{array}$ & $\begin{array}{l}-7.73 \\
(69.83)\end{array}$ \\
\hline Current ratio & $\begin{array}{l}1.70 \\
(1.64)\end{array}$ & $\begin{array}{l}2.37 \\
(2.27)\end{array}$ & $\begin{array}{l}1.44 \\
(1.43)\end{array}$ & $\begin{array}{l}1.73 \\
(1.67)\end{array}$ & $\begin{array}{l}1.76 \\
(1.62)\end{array}$ & $\begin{array}{l}1.79 \\
(1.58)\end{array}$ & $\begin{array}{l}1.65 \\
(1.07)\end{array}$ \\
\hline $\begin{array}{l}\text { Intensity of } \\
\text { investment }\end{array}$ & $\begin{array}{l}0.54 \\
(0.26)\end{array}$ & $\begin{array}{l}0.68 \\
(0.20)\end{array}$ & $\begin{array}{l}0.49 \\
(0.24)\end{array}$ & $\begin{array}{l}0.60 \\
(0.24)\end{array}$ & $\begin{array}{l}0.48 \\
(0.24)\end{array}$ & $\begin{array}{l}0.59 \\
(0.28)\end{array}$ & $\begin{array}{l}0.27 \\
(0.27)\end{array}$ \\
\hline $\begin{array}{l}\text { Asset intensity } \\
1\end{array}$ & $\begin{array}{l}3.63 \\
(4.61)\end{array}$ & $\begin{array}{l}5.97 \\
(4.66)\end{array}$ & $\begin{array}{l}2.72 \\
(3.78)\end{array}$ & $\begin{array}{l}4.40 \\
(5.10)\end{array}$ & $\begin{array}{l}2.18 \\
(3.07)\end{array}$ & $\begin{array}{l}6.38 \\
(6.64)\end{array}$ & $\begin{array}{l}0.83 \\
(1.45)\end{array}$ \\
\hline $\begin{array}{l}\text { Continuous } \\
\text { intensity }\end{array}$ & $\begin{array}{l}0.30 \\
(0.20)\end{array}$ & $\begin{array}{l}0.20 \\
(0.17)\end{array}$ & $\begin{array}{l}0.34 \\
(0.18)\end{array}$ & $\begin{array}{l}0.26 \\
(0.18)\end{array}$ & $\begin{array}{l}0.37 \\
(0.20)\end{array}$ & $\begin{array}{l}0.24 \\
(0.25)\end{array}$ & $\begin{array}{l}0.53 \\
(0.23)\end{array}$ \\
\hline Asset intensity 2 & $\begin{array}{l}1.37 \\
(2.93)\end{array}$ & $\begin{array}{l}0.49 \\
(1.00)\end{array}$ & $\begin{array}{l}1.32 \\
(2.39)\end{array}$ & $\begin{array}{l}0.99 \\
(2.35)\end{array}$ & $\begin{array}{l}2.06 \\
(4.19)\end{array}$ & $\begin{array}{l}0.95 \\
(1.90)\end{array}$ & $\begin{array}{l}5.22 \\
(4.50)\end{array}$ \\
\hline Asset turnover & $\begin{array}{l}0.75 \\
(0.63)\end{array}$ & $\begin{array}{l}0.55 \\
(0.34)\end{array}$ & $\begin{array}{l}0.99 \\
(0.70)\end{array}$ & $\begin{array}{l}0.51 \\
(0.46)\end{array}$ & $\begin{array}{l}1.02 \\
(0.62)\end{array}$ & $\begin{array}{l}0.51 \\
(0.50)\end{array}$ & $\begin{array}{l}1.65 \\
(0.84)\end{array}$ \\
\hline $\begin{array}{l}\text { Current asset } \\
\text { turnover }\end{array}$ & $\begin{array}{l}2.83 \\
(1.78)\end{array}$ & $\begin{array}{l}3.20 \\
(1.55)\end{array}$ & $\begin{array}{l}3.23 \\
(1.86)\end{array}$ & $\begin{array}{l}2.26 \\
(1.56)\end{array}$ & $\begin{array}{l}3.28 \\
(1.63)\end{array}$ & $\begin{array}{l}3.05 \\
(2.35)\end{array}$ & $\begin{array}{l}4.35 \\
(2.03)\end{array}$ \\
\hline $\begin{array}{l}\text { Debt to equity } \\
\text { ratio }\end{array}$ & $\begin{array}{l}1.18 \\
(2.79)\end{array}$ & $\begin{array}{l}1.21 \\
(1.88)\end{array}$ & $\begin{array}{l}0.79 \\
(2.07)\end{array}$ & $\begin{array}{l}1.21 \\
(2.67)\end{array}$ & $\begin{array}{l}1.72 \\
(3.77)\end{array}$ & $\begin{array}{l}0.95 \\
(3.99)\end{array}$ & $\begin{array}{l}0.87 \\
(2.09)\end{array}$ \\
\hline Equity ratio & $\begin{array}{l}0.64 \\
(0.51)\end{array}$ & $\begin{array}{l}0.57 \\
(0.26)\end{array}$ & $\begin{array}{l}0.71 \\
(0.33)\end{array}$ & $\begin{array}{l}0.63 \\
(0.60)\end{array}$ & $\begin{array}{l}0.54 \\
(0.56)\end{array}$ & $\begin{array}{l}0.61 \\
(0.29)\end{array}$ & $\begin{array}{l}0.81 \\
(0.25)\end{array}$ \\
\hline ROE & $\begin{array}{l}0.07 \\
(0.30)\end{array}$ & $\begin{array}{l}0.05 \\
(0.21)\end{array}$ & $\begin{array}{l}0.06 \\
(0.30)\end{array}$ & $\begin{array}{l}0.06 \\
(0.29)\end{array}$ & $\begin{array}{l}0.10 \\
(0.28)\end{array}$ & $\begin{array}{l}0.04 \\
(0.15)\end{array}$ & $\begin{array}{l}0.18 \\
(0.55)\end{array}$ \\
\hline ROA & $\begin{array}{l}0.02 \\
(0.09)\end{array}$ & $\begin{array}{l}0.03 \\
(0.05)\end{array}$ & $\begin{array}{l}0.02 \\
(0.10)\end{array}$ & $\begin{array}{l}0.02 \\
(0.10)\end{array}$ & $\begin{array}{l}0.03 \\
(0.07)\end{array}$ & $\begin{array}{l}0.03 \\
(0.05)\end{array}$ & $\begin{array}{l}0.04 \\
(0.08)\end{array}$ \\
\hline $\begin{array}{l}\text { Net profit } \\
\text { margin }\end{array}$ & $\begin{array}{l}0.03 \\
(0.34)\end{array}$ & $\begin{array}{l}0.06 \\
(0.17)\end{array}$ & $\begin{array}{l}0.02 \\
(0.30)\end{array}$ & $\begin{array}{l}0.03 \\
(0.45)\end{array}$ & $\begin{array}{l}0.02 \\
(0.13)\end{array}$ & $\begin{array}{l}0.04 \\
(0.14)\end{array}$ & $\begin{array}{l}0.04 \\
(0.07)\end{array}$ \\
\hline
\end{tabular}

variables) of these LSPs for the year 2010 are shown in Table 1.

- LSPs with the appropriate SIC code and activity since at least 2006 were chosen for the analysis of systematic risk and microeconomic variables. An active period from 2006 to 2010 was required in order to calculate beta. Values for beta (own calculation) and the appropriate microeconomic variables were available for 702 LSPs. The characteristics (microeconomic variables) of these LSPs (those shown in Table 1 are also included) for the year 2010 are shown in Table 2.

- For the regression analyses of $\mathrm{CoC}$ and macroeconomic variables, the period from 2006 to 2010 was analyzed: 226 LSPs. For the regression analyses of systematic risk and macroeconomic variables, the period from 2000 to 2010 was analyzed. Hence, only LSPs that have been active since at least 1996 (to calculate beta) were considered: 416 LSPs.

\subsection{Descriptive statistics}

No uniform financial structure of LSPs exists. The basic descriptive statistics of the analyzed microeconomic ratios for both analyzed samples, the largest with 702 LSPs for regression analyses with beta as the dependent variable as well as the abstracted smaller sample of 437 LSPs for regression analyses with WACC as the dependent variable, are shown in Tables 1 and 2. 
Table 3 Results of regression analyses of $\mathrm{CoC}$ and microeconomic variables

\begin{tabular}{|c|c|c|c|c|c|c|c|}
\hline & \multicolumn{7}{|c|}{ Dependent variable: WACC } \\
\hline & All LSPs & $\begin{array}{l}\text { Railroad } \\
\text { transportation } \\
\text { SIC } 40\end{array}$ & $\begin{array}{l}\text { Motor freight } \\
\text { transportation } \\
\text { SIC } 42\end{array}$ & $\begin{array}{l}\text { Water } \\
\text { transportation } \\
\text { SIC } 44\end{array}$ & $\begin{array}{l}\text { Transportation } \\
\text { by air } \\
\text { SIC } 45\end{array}$ & $\begin{array}{l}\text { Pipeline, except } \\
\text { natural gas } \\
\text { SIC } 46\end{array}$ & $\begin{array}{l}\text { Transportation } \\
\text { services } \\
\text { SIC } 47\end{array}$ \\
\hline $\begin{array}{l}\text { Number of } \\
\text { LSPs }\end{array}$ & 437 & 30 & 120 & 184 & 74 & 11 & 18 \\
\hline$R^{2}$ & 0.87 & 0.919 & 0.69 & 0.809 & 0.707 & 0.843 & 0.998 \\
\hline Ratios & \multicolumn{7}{|c|}{ Standardized slope of regression ( $t$ value in parentheses) } \\
\hline (Constant) & $-(3.295)$ & $-(1.716)$ & $(4.653)$ & $-(1.031)$ & $(1.997)$ & $-(0.311)$ & $(2.701)$ \\
\hline Current ratio & & & & & & $\begin{array}{l}-0.385^{* *} \\
-(2.461)\end{array}$ & $\begin{array}{r}-0.440 * * * \\
-(3.602)\end{array}$ \\
\hline $\begin{array}{l}\text { Intensity of } \\
\text { investment }\end{array}$ & $\begin{array}{l}0.046^{* *} \\
(2.285)\end{array}$ & & $\begin{array}{l}-0.125^{* *} \\
-(2.334)\end{array}$ & & & & \\
\hline $\begin{array}{l}\text { Continuous } \\
\text { intensity }\end{array}$ & & & & & & $\begin{array}{r}0.751 * * * \\
(4.841)\end{array}$ & \\
\hline Asset intensity 2 & & $\begin{array}{l}0.142 * * \\
\quad(2.273)\end{array}$ & & & & & \\
\hline Asset turnover & & & & $\begin{array}{r}0.155^{* * * *} \\
(3.289)\end{array}$ & $\begin{array}{l}-0.153 * * \\
-(2.227)\end{array}$ & & \\
\hline $\begin{array}{l}\text { Debt to equity } \\
\text { ratio }\end{array}$ & $\begin{array}{r}-0.114 * * * \\
-(4.281)\end{array}$ & & & & & & \\
\hline Equity ratio & $\begin{array}{r}0.163 * * * \\
(5.707)\end{array}$ & $\begin{array}{r}0.205^{* * * *} \\
(3.345)\end{array}$ & & & & & \\
\hline ROE & $\begin{array}{l}1.099 * * * \\
(49.845)\end{array}$ & $\begin{array}{l}0.865 * * * \\
(15.159)\end{array}$ & & $\begin{array}{c}0.265 * * * \\
(3.829)\end{array}$ & & & $\begin{array}{l}0.996 * * * \\
(80.132)\end{array}$ \\
\hline ROA & $\begin{array}{l}-0.357 * * * \\
-(15.525)\end{array}$ & & $\begin{array}{l}0.729 * * * \\
(13.617)\end{array}$ & $\begin{array}{r}0.655^{* * * *} \\
(9.629)\end{array}$ & $\begin{array}{r}0.741 * * * \\
(8.919)\end{array}$ & $\begin{array}{r}0.532 * * * \\
(3.509)\end{array}$ & $\begin{array}{r}-0.080^{* * * *} \\
-(6.504)\end{array}$ \\
\hline $\begin{array}{l}\text { Net profit } \\
\text { margin }\end{array}$ & & & & $\begin{array}{l}-0.115^{* *} \\
-(2.176)\end{array}$ & $\begin{array}{l}0.166^{* *} \\
(2.006)\end{array}$ & & \\
\hline Beta & $\begin{array}{r}-0.054 * * * \\
-(3.065)\end{array}$ & & $\begin{array}{r}-0.267 * * * \\
-(5.077)\end{array}$ & & & & $\begin{array}{l}-0.028 * * \\
-(2.251)\end{array}$ \\
\hline
\end{tabular}

*** Significant at $1 \%$ level; ** Significant at $5 \%$ level; * Significant at $1 \%$ level

Compared to other industries such as the retail or machinery industry, LSPs have a relatively low mean value for beta (0.31) (the retail industry is 1.01 in Europe and 1.0 in the USA; the machinery industry is 1.14 in Europe and 1.04 in the USA) and an average value for WACC (8.4 \%) (the retail industry is $8.3 \%$ in Europe and $6.9 \%$ in the USA; the machinery industry is $9.6 \%$ in Europe and $7.8 \%$ in the USA) [63]. Detailed statistics indicating absolute values are presented in the Tables 9, 10.

\section{Results}

\subsection{Regression of $\mathrm{CoC}$ and microeconomic variables}

The regression analysis of $\mathrm{CoC}$ and microeconomic variables (Table 3) showed a general set of variables that significantly influences the CoC of all LSPs.

These microeconomic variables are as follows: intensity of investment, debt to equity ratio, equity ratio, ROE, ROA, and beta. The overall explanatory power of the model (coefficient of determination, $R^{2}$ ) is very high $(0.87)$, which means that $87 \%$ of the variance could be explained by the appropriate microeconomic variables. Regarding the remaining LSP clusters (the industry sector in which the LSPs operate), some differences can be observed. Not all microeconomic variables influence the $\mathrm{CoC}$ of the different LSP clusters to the same extent. Nevertheless, all regression models show high explanatory power. For example, the current ratio is the only liquidity ratio that shows a significant influence on $\mathrm{CoC}$, but only in the Pipeline and Transportation Services cluster. All other ratios come under the asset, capital, or profitability structures.

\subsection{Regression of systematic risk and microeconomic variables}

Similar to the regression analysis of the effect of microeconomic variables on $\mathrm{CoC}$, the regression analysis of systematic risk and microeconomic variables reveals a set of variables significantly influencing the systematic risk of all LSPs (Table 4). 
Table 4 Results of regression analyses of systematic risk and microeconomic variables

\begin{tabular}{|c|c|c|c|c|c|c|c|}
\hline & \multicolumn{7}{|c|}{ Dependent variable: beta $(\beta)$} \\
\hline & All LSPs & $\begin{array}{l}\text { Railroad } \\
\text { transportation } \\
\text { SIC } 40\end{array}$ & $\begin{array}{l}\text { Motor freight } \\
\text { transportation } \\
\text { SIC } 42\end{array}$ & $\begin{array}{l}\text { Water } \\
\text { transportation } \\
\text { SIC } 44\end{array}$ & $\begin{array}{l}\text { Transportation } \\
\text { by air } \\
\text { SIC } 45\end{array}$ & $\begin{array}{l}\text { Pipeline, except } \\
\text { natural gas } \\
\text { SIC } 46\end{array}$ & $\begin{array}{l}\text { Transportation } \\
\text { services } \\
\text { SIC } 47\end{array}$ \\
\hline $\begin{array}{l}\text { Number of } \\
\text { LSPs }\end{array}$ & 702 & 43 & 164 & 321 & 131 & 20 & 23 \\
\hline$R^{2}$ & 0.034 & - & 0.03 & 0.077 & 0.044 & 0.874 & - \\
\hline Ratios & \multicolumn{7}{|c|}{ Standardized slope of regression ( $t$ value in parentheses) } \\
\hline (Constant) & $(10.458)$ & & $(0.602)$ & $(11.169)$ & $(6.445)$ & $(2.147)$ & \\
\hline Current ratio & & & & & & $0.433 * * *(4.523)$ & \\
\hline $\begin{array}{l}\text { Asset intensity } \\
1\end{array}$ & & & & & & $\begin{array}{l}-0.392 * * *- \\
(3.376)\end{array}$ & \\
\hline $\begin{array}{l}\text { Continuous } \\
\text { intensity }\end{array}$ & $\begin{array}{c}-0.193 * * *- \\
(4.055)\end{array}$ & & & & & & $\begin{array}{c}-0.278 * * *- \\
(5.171)\end{array}$ \\
\hline Asset turnover & $\begin{array}{c}0.132 * * * \\
(2.777)\end{array}$ & & & & & & \\
\hline $\begin{array}{l}\text { Current asset } \\
\text { turnover }\end{array}$ & & & $0.174 * *(2.245)$ & & & $0.731 * * *(6.470)$ & \\
\hline $\begin{array}{l}\text { Debt to equity } \\
\text { ratio }\end{array}$ & $\begin{array}{r}0.089 * * \\
(2.375)\end{array}$ & & & & $\begin{array}{l}0.209 * * \\
(2.426)\end{array}$ & & \\
\hline Equity ratio & & & & & & $\begin{array}{l}-0.311^{* *}- \\
(2.515)\end{array}$ & \\
\hline
\end{tabular}

*** Significant at $1 \%$ level; $* *$ Significant at $5 \%$ level; * Significant at $1 \%$ level

Table 5 Results of regression analyses of $\mathrm{CoC}$ and macroeconomic variables

\begin{tabular}{|c|c|c|c|c|c|c|c|}
\hline & \multicolumn{7}{|c|}{ Dependent variable: WACC, years 2006-2010 } \\
\hline & $\begin{array}{l}\text { All } \\
\text { LSPs }\end{array}$ & $\begin{array}{l}\text { Railroad } \\
\text { transportation } \\
\text { SIC } 40\end{array}$ & $\begin{array}{l}\text { Motor freight } \\
\text { transportation } \\
\text { SIC } 42\end{array}$ & $\begin{array}{l}\text { Water } \\
\text { transportation } \\
\text { SIC } 44\end{array}$ & $\begin{array}{l}\text { Transportation } \\
\text { by air } \\
\text { SIC } 45\end{array}$ & $\begin{array}{l}\text { Pipeline, except } \\
\text { natural gas } \\
\text { SIC } 46\end{array}$ & $\begin{array}{l}\text { Transportation } \\
\text { services } \\
\text { SIC } 47\end{array}$ \\
\hline Number of LSPs & 226 & 19 & 73 & 87 & 31 & 8 & 8 \\
\hline$R^{2}$ & - & 0.824 & - & 0.895 & 0.93 & - & 0.88 \\
\hline $\begin{array}{l}\text { Macroeconomic } \\
\text { variables }\end{array}$ & \multicolumn{7}{|c|}{ Standardized slope of regression ( $t$ value in parentheses) } \\
\hline Constant & & $(-3.499)$ & & $(5.086)$ & $(-4.506)$ & & $(-1.652)$ \\
\hline $\begin{array}{l}\text { Money supply (M2) } \\
\text { as \% of GDP }\end{array}$ & & $0.908 * *(3.744)$ & & & & & \\
\hline $\begin{array}{l}\text { Employment to } \\
\text { population ratio }\end{array}$ & & & & $\begin{array}{r}-0.946 * * \\
(-5.046)\end{array}$ & & & \\
\hline $\mathrm{CO}_{2}(\mathrm{kt})$ & & & & & $0.964 * * *(6.316)$ & & \\
\hline GDP & & & & & & & $0.938 * *(4.691)$ \\
\hline
\end{tabular}

*** Significant at $1 \%$ level; ** significant at $5 \%$ level; * significant at $1 \%$ level

These microeconomic variables are as follows: continuous intensity, asset turnover, and debt to equity ratio, regarding asset and capital structure. In contrast to the analyses of $\mathrm{CoC}$ and microeconomic variables, the model-as well as the models considering the single industry cluster-shows very low overall explanatory power $\left(R^{2}\right)$. While the systematic risk of the Railroad Transportation and Transportation Services clusters is not significantly influenced by the microeconomic variables, the other clusters show obvious differences. The Pipeline cluster shows particular differences. Its systematic risk is significantly influenced by four ratios: current ratio, asset intensity 1, current asset turnover, and equity ratio. Furthermore, the regression model is the only one that shows a very high explanatory power (0.874). 
Table 6 Results of regression analyses of systematic risk and macroeconomic variables

\begin{tabular}{|c|c|c|c|c|c|c|c|}
\hline & \multicolumn{7}{|c|}{ Dependent variable: beta, years 2000-2010 } \\
\hline & All LSPs & $\begin{array}{l}\text { Railroad } \\
\text { transportation } \\
\text { SIC } 40\end{array}$ & $\begin{array}{l}\text { Motor freight } \\
\text { transportation } \\
\text { SIC } 42\end{array}$ & $\begin{array}{l}\text { Water } \\
\text { transportation } \\
\text { SIC } 44\end{array}$ & $\begin{array}{l}\text { Transportation } \\
\text { by air } \\
\text { SIC } 45\end{array}$ & $\begin{array}{l}\text { Pipeline, except } \\
\text { natural gas } \\
\text { SIC } 46\end{array}$ & $\begin{array}{l}\text { Transportation } \\
\text { services } \\
\text { SIC } 47\end{array}$ \\
\hline Number of LSPs & 416 & 33 & 123 & 175 & 69 & 5 & 11 \\
\hline$R^{2}$ & 0.993 & 0.98 & 0.932 & 0.983 & 0.956 & 0.862 & 0.974 \\
\hline $\begin{array}{l}\text { Macroeconomic } \\
\text { variables }\end{array}$ & \multicolumn{7}{|c|}{ Standardized slope of regression ( $t$ value in parentheses) } \\
\hline Constant & $(33.006)$ & $(19.977)$ & $(15.583)$ & $(9.942)$ & (7.637) & $(8.150)$ & $(22.628)$ \\
\hline $\begin{array}{l}\text { Adjusted net national } \\
\text { income }\end{array}$ & $\begin{array}{r}-1.366 * * * \\
(-15.209)\end{array}$ & & & & & & \\
\hline Mean oil price & $\begin{array}{r}0.406^{* * * *} \\
(4.519)\end{array}$ & & & & & & \\
\hline $\mathrm{CO}_{2}(\mathrm{kt})$ & & $\begin{array}{r}-0.827 * * * \\
(-10.473)\end{array}$ & & $\begin{array}{r}-1.660 * * * \\
(-6.337)\end{array}$ & & $\begin{array}{r}-0.929 * * * \\
(-7.078)\end{array}$ & $\begin{array}{r}-0.987 * * * \\
(-17.176)\end{array}$ \\
\hline Market capitalization & & $\begin{array}{l}-0.208 * * \\
(-2.639)\end{array}$ & $\begin{array}{r}-0.966 * * * \\
(-10.51)\end{array}$ & & & & \\
\hline GNI & & & & $\begin{array}{l}0.690 * * \\
(2.632)\end{array}$ & & & \\
\hline $\begin{array}{l}\text { Money supply (M2) } \\
\text { as \% of GDP }\end{array}$ & & & & & $\begin{array}{r}-0.607 * * * \\
(-5.073)\end{array}$ & & \\
\hline $\begin{array}{l}\text { Gross capital } \\
\text { formation }\end{array}$ & & & & & $\begin{array}{r}-0.463 * * * \\
(-3.646)\end{array}$ & & \\
\hline
\end{tabular}

*** Significant at $1 \%$ level; $* *$ Significant at $5 \%$ level; * Significant at $1 \%$ level

\subsection{Regression of $\mathrm{CoC}$ and macroeconomic variables}

The regression analyses of $\mathrm{CoC}$ and macroeconomic variables (Table 5) do not reveal a general set of macroeconomic variables that influences the $\mathrm{CoC}$ of LSPs.

$\mathrm{CoC}$ is influenced by money supply (M2) in the Railroad Transportation cluster, by the employment to population ratio in the Water Transportation cluster, by $\mathrm{CO}_{2}$ in the Transportation by Air cluster, and by GDP in the Transportation Services cluster. The explanatory power of the models or variables (only one variable in each cluster was included) is nevertheless relatively high.

\subsection{Regression of systematic risk and macroeconomic variables}

In contrast to the results of the regression analyses of $\mathrm{CoC}$ and the macroeconomic variables, the regression analysis of systematic risk and the macroeconomic variables reveals a set of macroeconomic variables that influences the $\mathrm{CoC}$ of all LSPs (adjusted net national income and the mean oil price). Regarding the single industry clusters, $\mathrm{CO}_{2}$ emissions significantly influence the systematic risk of the Railroad and Water Transportation, Pipeline, and Transportation Services clusters. Market capitalization is relevant in the Railroad and Motor Freight Transportation clusters, and the Transportation by Air cluster is the only one where $\mathrm{CoC}$ is influenced by money supply and gross capital formation. The explanatory power $\left(R^{2}\right)$ of all models is very high in all clusters and is higher than $R^{2}$ of the analyses of $\mathrm{CoC}$ and macroeconomic values.

\section{Discussion}

\subsection{Discussion of industry characteristics}

Hypothesis $1 a$ and $1 b$ : The regression analyses showed significant differences between the different LSP groups, clustered according to SIC code and the predominant mode of transport (Tables 3, 4, 5, 6). Our results reveal no homogenous set of variables influencing the $\mathrm{CoC}$ or systematic risk of LSPs. The analysis of $\mathrm{CoC}$ and microeconomic variables indeed led to a set of variables influencing the CoC of all LSPs. Nevertheless, this set of variables was not valid for all industry clusters. A similar pattern was observed in the regression analyses of the systematic risk and microeconomic variables (Table 4) and of the systematic risk and macroeconomic variables. The results are in line with the findings of Kavussanos and Marcoulis [16, 44], who also showed variations in stock market perception (by applying the Capital Asset Pricing Model [CAPM]) and systematic risk of different LSP industries. Therefore, H1a and $\mathrm{H} 1 \mathrm{~b}$ are supported. 


\subsection{Discussion of company characteristics}

Hypothesis 2a: The results indicated that a variety of microeconomic variables influence the $\mathrm{CoC}$ of all analyzed LSPs: debt to equity ratio, equity ratio, intensity of investment, ROE, ROA, and systematic risk.

- The influence of debt to equity ratio and equity ratio is obvious because the factors of debt and equity have been included in the calculation of WACC. The equity ratio showed a positive influence on WACC, meaning that the more LSPs are funded by equity, the higher their CoC. Consequently, the debt to equity ratio had a negative influence on WACC, which implies that CoC decreases when LSPs strengthen their debt funding (up to a certain point). Because all analyzed LSPs show a relatively high equity ratio (Table 1), they could take advantage of better credit conditions (while interest payments are tax exempt), which would lead to lower CoC [64]. This result supports Modigliani and Miller's [65] classic theorem on cost of capital. An "optimal" debt to equity ratio minimizes a company's $\mathrm{CoC}$, but $\mathrm{CoC}$ also increases if the equity ratio exceeds a specific barrier.

- The intensity of investment showed a positive influence on the WACC of all LSPs, indicating that a higher intensity of investment leads to higher CoC. All LSP clusters, except Transportation Services, are-more or less-asset based (Table 1), which means that they have several tangible resources (property, plants, and equipment), and their capital is tied up and not available on short notice. Hence, the asset flexibility of LSPs (except for Transportation Services) is low, which is also indicated by the intensity of investment; the higher this ratio, the lower the asset flexibility, and consequently, the higher the $\mathrm{CoC}$, which is also partly shown by Sudarsanam [47].

- The variables of ROE and ROA are related to the profitability structure of LSPs. ROE and ROA are performance indicators that indicate whether the operation of an LSP is profitable [66]. Similar to the equity ratio, ROE had a positive influence on WACC, which means that the more that LSPs are funded by equity, the higher their CoC. This outcome supports the results of Jung [67], who also showed a positive influence of $\mathrm{ROE}$ on CoC. The fact that the CoC of LSPs decreases if they strengthen their debt funding must not be confounded with a relatively high equity ratio, which may lead to better credit conditions at the same time. The equity ratio does not imply that all equity is used for investments. On the contrary, ROA had a negative influence on WACC, meaning the more profitable an LSP operates in regard to assets invested, the lower its CoC.
- Surprisingly, systematic risk also showed a negative influence on CoC. A positive influence was expected. This result could be ascribed to the methodology used to calculate WACC. ${ }^{3}$ The influence was negative in combination with the influence of the other microeconomic variables. Furthermore, the standardized slope of the regression showed a low value comparison with the slope of the other significant ratios. The results indicate that there seems to be an "optimal" value for the systematic risk of LSPs, minimizing their CoC. This assumption requires further validation in future research.

Regarding the single industry clusters of LSPs, no clusters showed the same set of variables influencing CoC:

- Railroad Transportation: The WACC of the Railroad Transportation cluster was positively influenced by asset intensity 2, equity ratio, and ROE. Hence, for this non-current, asset-based cluster, lower non-current assets or higher current assets would lead to higher CoC. Furthermore, WACC increased with higher equity ratio and hence ROE. This result could be ascribed to the fact that railroad companies are characterized by the lowest mean equity ratio and the highest debt to equity ratio. A rise in equity would fundamentally change the (optimal) financial structure of this cluster and lead to increasing $\mathrm{CoC}$ (and vice versa).

- Motor Freight Transportation: The WACC of the Motor Freight Transportation cluster was negatively influenced by intensity of investment and systematic risk and positively influenced by ROA. The Motor Freight companies show relatively low asset intensity 1 . If, in the case of the Motor Freight Transportation cluster, LSPs' share of non-current assets decreased (intensity of investment), CoC would then increase. A certain amount of non-current assets is inevitable to ensure the operation of an LSP. As described for all LSPs, systematic risk also negatively influenced $\mathrm{CoC}$ in this cluster. The positive influence of ROA on WACC is surprising but, compared with the other clusters, it could be ascribed to the "light" asset structure of LSPs in the Motor Freight Transportation cluster.

- Water Transportation: The WACC of the Water Transportation cluster was, in particular, negatively influenced by net profit margin and positively influenced by asset turnover and ROA. Regarding the net profit margin of LSPs in this cluster, the more profitably they operate, the lower their CoC. The effect of ROA could be ascribed to the capital and asset structure of

\footnotetext{
3 Alternative approaches for calculating $\mathrm{CoC}$ are the discounted cash flow method (DCF), the arbitrage pricing theory (APT), and consumption-based models [31, 32].
} 
this cluster. On the one hand, the cluster shows the lowest mean value for asset and current asset turnover, indicating the ratio of annual revenues and total or current assets, respectively. On the other hand, the cluster reveals the highest mean net profit margin, which could explain the negative relationship.

- Transportation by Air: The WACC of the Transportation by Air cluster was negatively influenced by asset turnover and positively influenced by ROA and net profit margin. The results are somewhat surprising; the higher the profitability of this cluster, the higher the CoC. This result cannot be explained and warrants further investigation.

- Pipelines except Natural Gas: The WACC of the Pipeline cluster was negatively influenced by the current ratio and positively influenced by continuous intensity and ROA. The ability to pay current liabilities (indicated by a current ratio of approximately $150 \%$ [66]) showed major significance for this cluster, negatively influencing its WACC. The higher the ratio of current assets to total assets, the higher the Pipeline cluster's CoC. The cluster shows the lowest mean values for asset intensity 2 and asset turnover.

- Transportation Services: The WACC of the Transportation Services cluster was negatively influenced by current ratio, $\mathrm{ROA}$, and systematic risk and positively influenced by ROE. In this cluster, the ability to pay current liabilities was also significant for $\mathrm{CoC}$. The asset structure of this cluster sharply differs from the other clusters (low level of fixed assets), which may explain the negative influence of ROA on WACC. The positive influence of ROE was obvious because this cluster reveals the highest mean value of the equity ratio. The results showed that $\mathrm{CoC}$ increased if the equity ratio exceeded a specific barrier.

The comparison of the LSP clusters indicates only a few similarities, which underscores the general outcomes of the research of Hofmann and Lampe [21] on the differences of the financial structure of several LSP clusters. The current ratio negatively influenced the WACC of the Pipeline and the Transportation Services clusters. Although both clusters have a "healthy" value for their ability to pay current liabilities, the results showed that this ratio was of major importance for their CoC. Furthermore, the ROE positively influenced the WACC of the Railroad and Water Transportation and the Transportation Service clusters. This result demonstrates the strong influence of this ratio on the $\mathrm{CoC}$ of LSPs. The ROA had a positive influence on all WACCs of all LSP clusters, except the Transportation Services cluster. This result could be ascribed to the completely different asset structures of the analyzed clusters (from "full-assetbased" to "asset-light"). Hypothesis 2a is supported.
Hypothesis $2 b$ : The results of the regression analyses of the systematic risk and microeconomic variables (Table 4) revealed that some variables significantly influenced the systemic risk of LSPs. This generally supports recent research results [e.g., 37, 68]. However, regarding the explanatory power of all clusters, $R^{2}$ was somewhat low. Therefore, a detailed discussion of the influence of each variable is not included in this discussion.

As the discussion shows, the influence of microeconomic variables on $\mathrm{CoC}$ is more significant than on systematic risk. Recent literature focused either on the influence on CoC or systematic risk, but did not compare both relationships. Hypothesis $2 \mathrm{~b}$ is supported.

\subsection{Discussion of market characteristics}

Hypothesis $3 a$ : The results showed that a variety of macroeconomic variables influenced the systematic risk of LSPs. The systematic risk of all LSPs was negatively influenced by adjusted national income and positively by the mean oil price. The latter relationship supports the findings of Arfaoui and Abaoub [39], who also revealed a positive influence of the mean oil price on systematic risk. Consequently, the higher the performance of the national economy is, the lower the systematic risk of LSPs.

Regarding the macroeconomic variables, we obtained the following results for each LSP cluster:

- Railroad Transportation: This cluster's systematic risk was negatively influenced by $\mathrm{CO}_{2}$ emissions and market capitalization. The latter relationship contradicts the findings of Patro et al. [69], who revealed a positive influence of market capitalization on systematic risk in general. The negative influence of $\mathrm{CO}_{2}$ emissions could be ascribed to the fact that decreased $\mathrm{CO}_{2}$ emissions imply reduced transports and hence poor market development for LSPs. Market capitalization can also be seen as a national economic indicator. The lower the market value of all listed companies, the higher the systematic risk. As the global economy strives for $\mathrm{CO}_{2}$ reduction and emissions trading becomes more established, future correlations may look different. High or increasing $\mathrm{CO}_{2}$ emissions might then imply high costs for LSPs (particularly in $\mathrm{CO}_{2^{-}}$ intensive industries like the Motor Freight Transportation cluster) and could possibly come along with a higher systematic risk.

- Motor Freight Transportation: This cluster's systematic risk (average 0.18) was negatively influenced only by market capitalization, which also contradicts the findings of Patro et al. [69]. The result indicates the (1) lowrisk affinity but (2) high dependency of this cluster on the development of the overall economy. The services 
are primarily demanded for short distance transports directly to or from the customer, that is, at the end or the beginning of a transport chain.

- Water Transportation: This cluster's systematic risk was negatively influenced by $\mathrm{CO}_{2}$ emissions and positively influenced by GNI, which is also partly shown by the analyses of Patro et al. [69]. The influence of $\mathrm{CO}_{2}$ emissions compensates for the influence of GNI.

- Transportation by Air: Most notably, the cluster's systematic risk was negatively influenced by money supply and gross capital formation. Both relationships are also supported by the findings of Mohammad and Hussain [70]. In addition to inflationary or deflationary developments, a higher money supply could also imply more economic activities and hence lead to decreasing systematic risk for LSPs. The same could be implied by gross capital formation.

- Pipeline except Natural Gas: This cluster's systematic risk was negatively influenced only by $\mathrm{CO}_{2}$ emissions, which seems to be the most important indicator for the development of the transportation services market.

- Transportation Services: This cluster's systematic risk was also negatively influenced by $\mathrm{CO}_{2}$ emissions.

Our analysis highlights a few common macroeconomic influencing factors. $\mathrm{CO}_{2}$ emissions had the highest influence on the systematic risk of LSPs. Recent research [71, 72] has made preliminary efforts to analyze the effect of $\mathrm{CO}_{2}$ emissions on companies' performance, but did not specifically investigate its direct influence on systematic risk or $\mathrm{CoC}$. This variable seems to be the most important indicator for the development of the transportation services market. Hypothesis $3 \mathrm{a}$ is supported.

Hypothesis 3b: The regression analyses of $\mathrm{CoC}$ and macroeconomic variables (Table 5) led to some significant results; however, in contrast to the influence of macroeconomic variables on systematic risk, no uniform set of variables influenced the $\mathrm{CoC}$ of all LSPs. Because the CoC of only four clusters was influenced by one macroeconomic variable, a detailed discussion is not provided.

As this discussion highlights, the influence of macroeconomic variables on systematic risk is more significant than on CoC. Recent literature focused either on the influence on $\mathrm{CoC}$ or systematic risk, but did not compare both relationships. Hypothesis $3 \mathrm{~b}$ is supported.

\subsection{Further findings}

In addition to the insights with regard to the hypotheses, we made two interesting additional findings: (a) regarding the influence of the LSPs' headquarters location on their systematic risk and $\mathrm{CoC}$; and (b) regarding the coherence between the systematic risk and CoC of LSPs.

a. The regression analyses of microeconomic and macroeconomic variables and WACC as well as beta (systematic risk), clustering LSPs according to their headquarters' locations, did not lead to notably significant results. Thus, we concluded that the country in which an LSP's headquarters is located has minor importance in explaining the $\mathrm{CoC}$ and systematic risk of LSPs. The results confirm the adequacy of the "territorial principle," that is, the country in which the LSP operates is crucial, not the flag under which a company "sails" ("nationality principle"). This finding is especially valid for globally linking LSPs, such as those allocated primarily to the Railroad or Water Transportation, Transportation by Air, and Pipeline clusters. This finding is supported by recent financial research revealing the increasing importance of industry factors in contrast to country locations [25, 33, 34, 50, 60].

b. Expected return on equity $\left(r_{\mathrm{e}}\right)$ is obligatory in calculating CoC, as follows: $r_{\mathrm{e}}=r_{\mathrm{f}}+\beta \cdot\left(r_{\mathrm{m}}-r_{\mathrm{f}}\right)$, where $r_{\mathrm{f}}$ is the risk-free interest rate and $r_{\mathrm{m}}$ is the expected return on the market portfolio. Based on this connectedness, we assumed the direct influence of systematic risk on the CoC of LSPs. However, comparison of the standardized slope of the regression showed that the influence was not as strong as that of the other significant microeconomic variables (Table 3). Although a positive correlation was expected, the correlation was negative. This unexpected finding could be ascribed to (1) the general low level of the whole LSP peer group's beta (0.31 on average) or (2) the method of calculating $\mathrm{CoC}$ in terms of WACC within the CAPM. The variable beta (systematic risk) is not necessary in all methods of calculating $\mathrm{CoC}$ [32], such as the arbitrage pricing theory (APT) [73] and the Fama-French, three-factor model [74], which is a limitation of this research.

\subsection{Limitations of the research}

First, only quoted LSPs were considered in the analyses because in calculating the $\mathrm{CoC}$ or systematic risk of an LSP, a variety of financial information is necessary, but was unavailable for unquoted LSPs. In practice, a common approach is to estimate the CoC of unquoted LSPs via benchmarks of similar LSPs regarding financial structure and field of activity [75]. Based on this common approach, we expect no significant differences when analyzing the CoC of non-quoted LSPs.

Second, the WACC approach within the context of the CAPM was used to calculate CoC. Alternative approaches 
for calculating $\mathrm{CoC}$ are the DCF method, the APT, and consumption-based models [31, 32]. The variable of systematic risk is not necessary in all methods used to calculate CoC [32], such as the APT [73] and the FamaFrench, three-factor model [74]. Although several studies have analyzed the differences in different methods used for calculating CoC, the results were not in agreement [76]. Surprisingly, our results showed that systematic risk negatively influenced the CoC of LSPs. Perhaps the importance of systematic risk in the CoC of LSPs is not very high. Further analyses applying alternative methods for calculating CoC could prove this assumption.

Third, the S\&P 500 market index was chosen as a reference for calculating beta. Because stock market indices often correlate [77], we do not expect significant differences when using another market index for calculating beta.

Fourth, $\mathrm{CoC}$ is an important consideration in decisions about how to invest capital, particularly the best strategy to follow [75]. Hence, it is a future-oriented variable. We analyzed the influence of micro- and macroeconomic variables, which are contingency factors oriented to the past, on CoC. In our analyses, we intended to identify the influence of these variables on $\mathrm{CoC}$, not to predict the development of the CoC of LSPs (this also verifies the application of a stepwise multiple linear regression). Nonetheless, the results allow conclusions regarding which significantly influential variables should be considered when analyzing the CoC of LSPs.

Fifth, even if we take a broad understanding of LSPs to include carriers as well as 3PL and 4PL LSPs, this widely adopted distinction of LSPs is not met by the SIC. This fact does not influence the results; nevertheless, future research could consider other clusters.

Sixth, our analysis was restricted to LSPs only. In order to fully understand similarities and differences of LSPs in comparison with other service providers as well as shipping, manufacturing, and retail companies, a cross-industry study is necessary.

\subsection{Theoretical contribution}

In summary, we made the following theoretical contributions:

1. Due to the very broad scope of our study, we built the first comprehensive bridge between logistics research (especially LSP-oriented studies) on the one hand and financial research (especially CoC-oriented studies) on the other hand. By showing statistical significances between contingency variables on the $\mathrm{CoC}$ and systematic risk of LSPs, we slightly enhanced former logistics research practices with (methodological) insights from finance.
2. Our second contribution is a "look inside the black box" of LSPs' CoC by working out relevant contingency variables and their influences. Astonishingly, both industry (predominant mode of transport) and company characteristics have very diverging impacts on the CoC of LSPs. This insight underscores the requirement to differentiate LSPs into homogenous subgroups (clusters). In order to compare "apples with apples," LSPs should not be lumped together in upcoming research investigations. In future empirical studies, it is recommended to not only use revenues or EBIT-margins as financial performance measures, but also CoC (e.g., WACC) and systematic risk as dependent variables.

3. Our results give valuable information about the risk profiles of LSPs as well as the measures taken by the LSPs to cope with market developments. Every type of LSP (according to predominant mode of transport) appears to have its own pattern of sensitivities regarding the different macroeconomic variables, which means that the $\mathrm{CoC}$ as well as the returns of each cluster have their own pattern of sensitivities to different market developments. Therefore, our findings can be used in defining a "risk-adequate" portfolio investment strategy. By changing the mix of stocks of different transportation industries, the amount and type of the risk exposures can be changed. Hence, a portfolio manager can define the risk exposure from each macroeconomic variable - as a risk factor-and therefore effectively diversify portfolios. For example, if an investor wants to reduce his risk exposure toward an increasing oil price, our results recommend to reduce stocks of air transportation and trucking companies. Furthermore, he could profit from a stronger investment in railroad transportation stocks.

4. Our results imply that not only the financial structure of LSPs, but also the market environment in which they are operating, significantly influence their $\mathrm{CoC}$ or systematic risk, respectively. CoC is an important criterion in strategic decisions that, e.g., concern future investments or M\&As and cooperation, as CoC allows for an assessment of potential takeover targets. The results of this study provide the first implications regarding how $\mathrm{CoC}$ and also the related systematic risk vary in different LSP industries and to what extent they are influenced by market and company characteristics. Thus, the results can facilitate the assessment of an LSP's competitors' performance or profitability. LSPs can also estimate to what extent their $\mathrm{CoC}$ and systematic risk will change if specific strategic decisions are taken and the appropriate market or industry characteristics are known. These insights are also helpful if strategic decisions are related with 
investment decisions, e.g., conclusions on $\mathrm{CoC}$ and systematic risk can be drawn when investing within a specific market or industry.

5. Although our analyses focused on quoted LSPs, nonquoted LSPs may also benefit from these insights. It is common to use data of quoted LSPs to estimate the CoC and systematic risk of non-quoted LSPs. Hence, non-quoted LSPs could compare their company-specific data with the results of this study in order to estimate their $\mathrm{CoC}$ as well as systematic risk. They then can identify potential for optimization (e.g., concerning their asset structure). Furthermore, if nonquoted LSPs have determined their CoC and systematic risk by the application of benchmarks, they may use this information for the same strategic decisions as quoted LSPs, e.g., concerning M\&As or cooperation as well as investment decisions. Moreover, they can also estimate the influence of specific strategic decisions, e.g., expanding into a new market, on their CoC.

\section{Conclusion and outlook}

We investigated $\mathrm{CoC}$ and systematic risk from the perspective of LSPs. CoC is an important consideration in strategic decisions of LSPs, in which financial information has become more and more important. Due to the importance of systematic risk as a key component of $\mathrm{CoC}$ (if calculated using WACC), the influence of the company, industry, and market characteristics on both $\mathrm{CoC}$ and systematic risk was analyzed. The main results answer the research question and are as follows:

- From an investor's point of view, the LSP business is rather nonvolatile. With an average beta of 0.31 , the whole logistics service provider industry reacts slower to market changes than the average of other industries. While this may be valued by risk-averse investors, major chances for high returns are somewhat rare.

- $\mathrm{CoC}$ and systematic risk significantly differ among the different LSP groups, clustered according the predominant mode of transport (SIC code). The industry clusters show several differences in the financial structures of LSPs.

- The CoC of LSPs is significantly influenced by microeconomic characteristics, which are companyspecific contingency variables, while macroeconomic variables do not show a significant influence. In particular, the asset and profitability structure of LSPs explains the close correlation with the CoC of LSPs (Table 7).
- The systematic risk of LSPs is significantly influenced by macroeconomic developments, which are marketspecific contingency variables, while the influence of microeconomic variables is lower. In particular, the amount of $\mathrm{CO}_{2}$ emissions seems to be an important indicator for the market development of LSPs and their systematic risk (Table 8).

- As a first additional insight, we found that the country in which an LSP's headquarters is located has no significant influence on the LSP's CoC. This fact could also be ascribed to the "territorial principle," which holds that the country (or countries) in which the LSP operates is crucial, but not the country in which the LSP's headquarters is located ("nationality principle").

- Finally, as a second additional finding, our study elaborated a negative influence of systematic risk on CoC. This result could be ascribed to limitations such as the methodology used to calculate CoC.

The main implication for management is the specific interdependencies of strategic decision making and $\mathrm{CoC}$. Particularly, in the logistics service provider industry, financial issues are often limited to sales profit margin and cost figures, thus neglecting the scope of other factors.

Much more theoretical and empirical work is needed to adequately develop financial insights in logistics research and in the LSP domain.

First, it is of interest to provide detailed analyses of similarities or differences between LSPs and other (service) industries regarding $\mathrm{CoC}$ and their influencing contingency variables. Such cross-industry studies might emphasize the characteristics of LSPs considered from a corporate finance perspective.

Furthermore, the regression analysis can be conducted using different regression techniques such as the stepwiseor forward-method, which may alter the results. Additionally, conducting analyses by using different methods of calculating the CoC of LSPs (especially methods that do not include the systematic risk-beta-as a variable) would be of interest.

Another goal might be to improve the homogeneity of the analyzed clusters. In our analysis, the LSPs were assigned according to their primary SIC code. As LSPs often operate in more than one SIC cluster, the assignment regarding their primary SIC code leads to clusters that are not $100 \%$ homogenous. Further studies might only analyze LSPs that operate in the area of one single SIC code to get homogeneous LSP clusters.

Although we do not expect significant differences when analyzing non-quoted LSPs, a case study or survey-based research analyzing the $\mathrm{CoC}$ of non-quoted LSPs could 


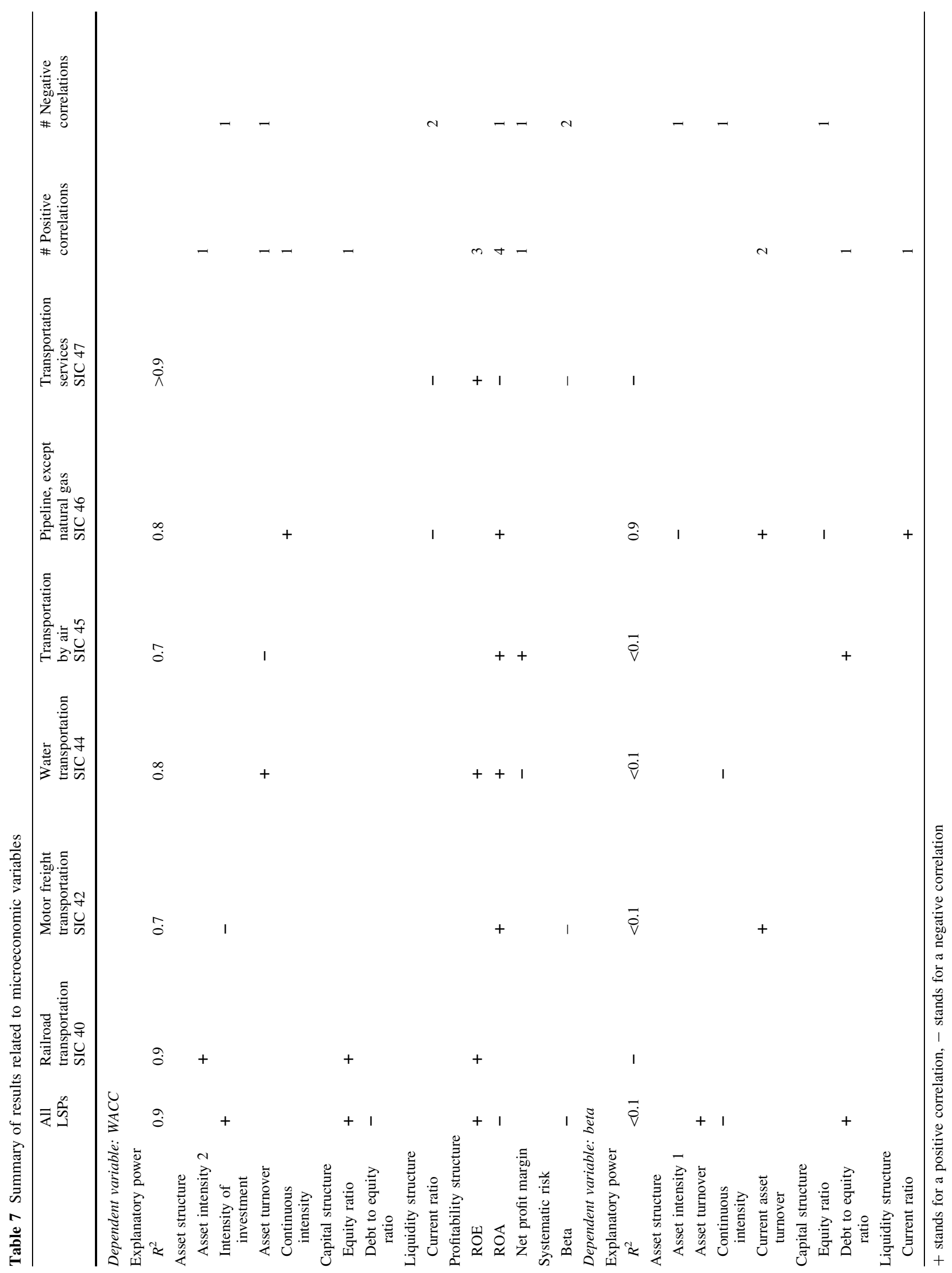


Logist. Res. (2014) 7:119

Page 19 of $25 \quad 119$

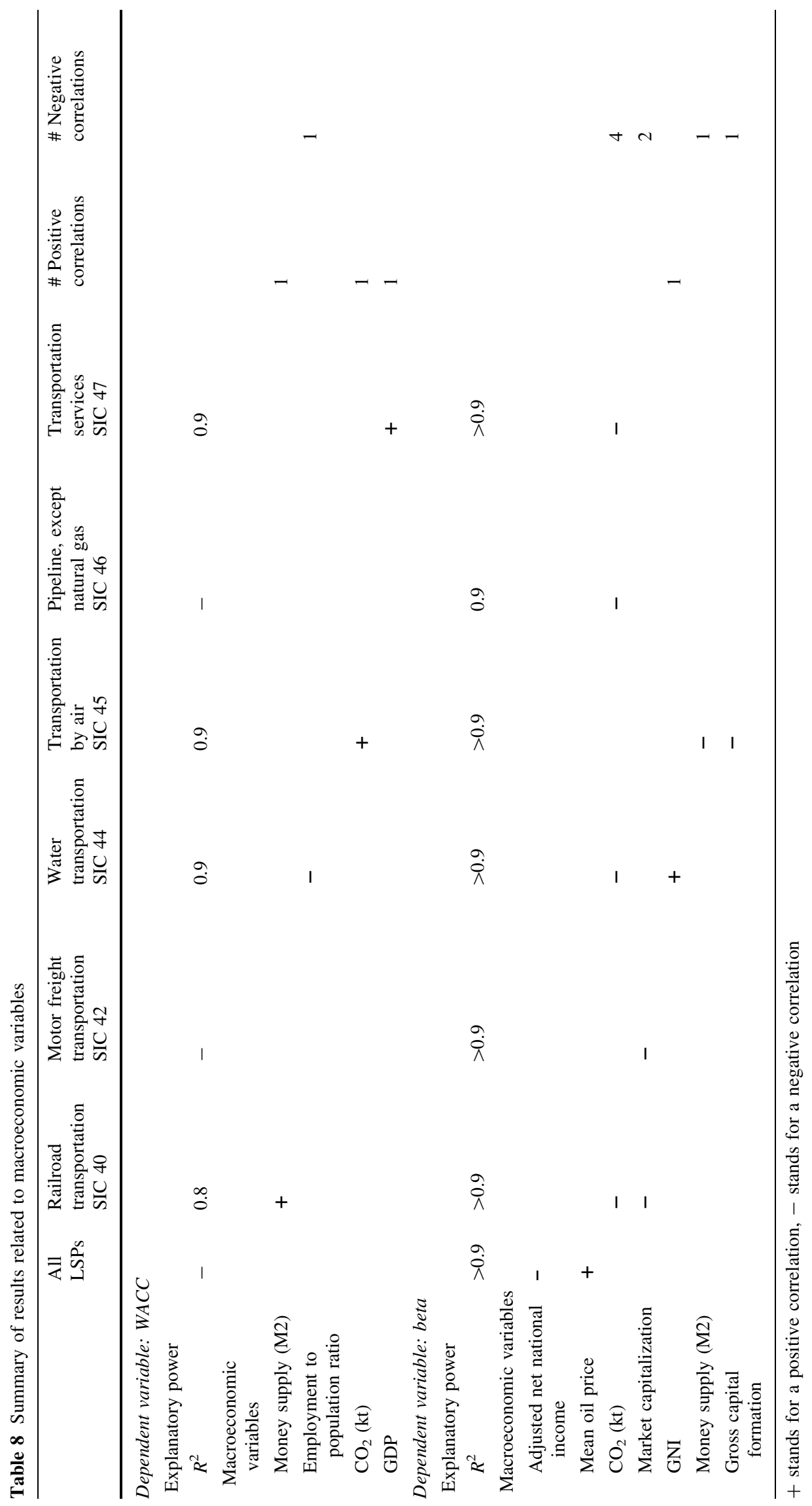

刑 Springer 
prove this assumption. Hence, a "good-practice" methodology for LSPs could be developed, and the partially answered question of the influence of systematic risk on $\mathrm{CoC}$ could perhaps be resolved.

In addition, empirical field research that focused on the relation between strategic decisions of LSPs and the consideration of $\mathrm{CoC}$ in these decisions could lead to interesting LSP-specific insights. In combination with an analysis of how rating agencies evaluate LSPs, the research recommended here could lead to an (financial) evaluation model specific to LSPs.

Generally, based on our broad approach that provides the first comprehensive overview on the determinants of LSPs' $\mathrm{CoC}$ and systematic risk, future research should now focus on more specific analyses.
Acknowledgments We would like to thank the two anonymous reviewers as well as the LORE-editor Herbert Kotzab for the valuable and constructive feedback. Further, the authors gratefully acknowledge the valuable comments on earlier versions of the paper from the participants of the NOFOMA 2013 Conference in Gothenburg, Sweden. The analyses of this paper are taken out of Kerstin Lampe's cumulative doctoral thesis.

Open Access This article is distributed under the terms of the Creative Commons Attribution License which permits any use, distribution, and reproduction in any medium, provided the original author(s) and the source are credited.

\section{Appendix}

See Tables 9, 10, 11, 12.

Table 9 Key financial figures (absolute) of logistics service providers used for regression analyses with WACC as dependent variable

\begin{tabular}{|c|c|c|c|c|c|c|c|}
\hline Cluster description & & $\begin{array}{l}\text { Railroad } \\
\text { transportation }\end{array}$ & $\begin{array}{l}\text { Motor freight } \\
\text { transportation }\end{array}$ & $\begin{array}{l}\text { Water } \\
\text { transportation }\end{array}$ & $\begin{array}{l}\text { Transportation } \\
\text { by air }\end{array}$ & $\begin{array}{l}\text { Pipeline, except } \\
\text { natural gas }\end{array}$ & $\begin{array}{l}\text { Transportation } \\
\text { services }\end{array}$ \\
\hline Cluster (SIC code) & ALL LSPs & SIC 40 & SIC 42 & SIC 44 & SIC 45 & SIC 46 & SIC 47 \\
\hline Number of LSPs & 437 & 32 & 130 & 189 & 78 & 12 & 20 \\
\hline Absolute (US\$) & \multicolumn{7}{|c|}{ Mean value (standard deviation in parentheses) } \\
\hline $\begin{array}{l}\text { Cash flow per } \\
\text { share }\end{array}$ & $\begin{array}{l}11.88 \\
(127.05)\end{array}$ & $\begin{array}{l}9.74 \\
(22.37)\end{array}$ & $\begin{array}{l}4.76 \\
(26.89)\end{array}$ & $\begin{array}{l}17.53 \\
(182.23)\end{array}$ & $\begin{array}{l}1.97 \\
(3.36)\end{array}$ & $\begin{array}{l}85.12 \\
(270.49)\end{array}$ & $\begin{array}{l}0.88 \\
(1.26)\end{array}$ \\
\hline $\begin{array}{l}\text { Total current } \\
\text { assets }\end{array}$ & $\begin{array}{l}682^{\prime} 548 \\
\left.1^{\prime} 728^{\prime} 497\right)\end{array}$ & $\begin{array}{l}991^{\prime} 216 \\
\left(1^{\prime} 348^{\prime} 184\right)\end{array}$ & $\begin{array}{l}267^{\prime} 256 \\
\left(610^{\prime} 315\right)\end{array}$ & $\begin{array}{l}521^{\prime} 210 \\
\left(1^{\prime} 431^{\prime} 305\right)\end{array}$ & $\begin{array}{l}1^{\prime} 551^{\prime} 589 \\
\left(2^{\prime} 781^{\prime} 161\right)\end{array}$ & $\begin{array}{l}1^{\prime} 481^{\prime} 335 \\
\left(3^{\prime} 752^{\prime} 412\right)\end{array}$ & $\begin{array}{l}545^{\prime} 411 \\
\left(763^{\prime} 291\right)\end{array}$ \\
\hline $\begin{array}{c}\text { Total current } \\
\text { liabilities }\end{array}$ & $\begin{array}{l}597^{\prime} 972 \\
\left(1^{\prime} 631^{\prime} 135\right)\end{array}$ & $\begin{array}{l}1^{\prime} 394^{\prime} 112 \\
\left(2^{\prime} 605^{\prime} 827\right)\end{array}$ & $\begin{array}{l}222^{\prime} 152 \\
\left(505^{\prime} 617\right)\end{array}$ & $\begin{array}{l}359^{\prime} 395 \\
\left(1^{\prime} 110^{\prime} 059\right)\end{array}$ & $\begin{array}{l}1^{\prime} 548^{\prime} 504 \\
\left(2^{\prime} 788^{\prime} 790\right)\end{array}$ & $\begin{array}{l}564^{\prime} 038 \\
\left(1^{\prime} 052^{\prime} 075\right)\end{array}$ & $\begin{array}{l}360^{\prime} 704 \\
\left(533^{\prime} 985\right)\end{array}$ \\
\hline EBIT & $\begin{array}{l}231^{\prime} 398 \\
\left(800^{\prime} 431\right)\end{array}$ & $\begin{array}{l}791^{\prime} 722 \\
\left(1^{\prime} 331^{\prime} 242\right)\end{array}$ & $\begin{array}{l}48^{\prime} 929 \\
\left(80^{\prime} 829\right)\end{array}$ & $\begin{array}{l}170^{\prime} 711 \\
\left(804^{\prime} 435\right)\end{array}$ & $\begin{array}{l}427^{\prime} 093 \\
\left(890^{\prime} 772\right)\end{array}$ & $\begin{array}{l}620^{\prime} 671 \\
\left(1^{\prime} 641^{\prime} 845\right)\end{array}$ & $\begin{array}{l}95^{\prime} 258 \\
\left(174^{\prime} 311\right)\end{array}$ \\
\hline EBITDA & $\begin{array}{l}356^{\prime} 620 \\
\left(1^{\prime} 190^{\prime} 820\right)\end{array}$ & $\begin{array}{l}1^{\prime} 232^{\prime} 314 \\
\left(2^{\prime} 069^{\prime} 551\right)\end{array}$ & $\begin{array}{l}82^{\prime} 227 \\
\left(136^{\prime} 233\right)\end{array}$ & $\begin{array}{l}257^{\prime} 116 \\
\left(1^{\prime} 202^{\prime} 655\right)\end{array}$ & $\begin{array}{l}675^{\prime} 109 \\
\left(1^{\prime} 278^{\prime} 491\right)\end{array}$ & $\begin{array}{l}858^{\prime} 553 \\
\left(2^{\prime} 293^{\prime} 070\right)\end{array}$ & $\begin{array}{l}122^{\prime} 382 \\
\left(192^{\prime} 264\right)\end{array}$ \\
\hline Long term debt & $\begin{array}{l}896^{\prime} 831 \\
\left(2^{\prime} 922^{\prime} 373\right)\end{array}$ & $\begin{array}{l}3^{\prime} 864^{\prime} 981 \\
\left(8^{\prime} 180^{\prime} 265\right)\end{array}$ & $\begin{array}{l}154^{\prime} 824 \\
\left(294^{\prime} 732\right)\end{array}$ & $\begin{array}{l}650^{\prime} 100 \\
\left(1^{\prime} 639^{\prime} 072\right)\end{array}$ & $\begin{array}{l}1^{\prime} 532^{\prime} 985 \\
\left(2^{\prime} 559^{\prime} 488\right)\end{array}$ & $\begin{array}{l}2^{\prime} 207^{\prime} 659 \\
\left(5^{\prime} 140^{\prime} 766\right)\end{array}$ & $\begin{array}{l}49^{\prime} 133 \\
\left(86^{\prime} 143\right)\end{array}$ \\
\hline Net income & $\begin{array}{l}134^{\prime} 728 \\
\left(445^{\prime} 570\right)\end{array}$ & $\begin{array}{l}386^{\prime} 382 \\
\left(683^{\prime} 963\right)\end{array}$ & $\begin{array}{l}28^{\prime} 797 \\
\left(60^{\prime} 526\right)\end{array}$ & $\begin{array}{l}99^{\prime} 044 \\
\left(370^{\prime} 261\right)\end{array}$ & $\begin{array}{l}271^{\prime} 204 \\
\left(617^{\prime} 791\right)\end{array}$ & $\begin{array}{l}415^{\prime} 383 \\
\left(1^{\prime} 075^{\prime} 462\right)\end{array}$ & $\begin{array}{l}56^{\prime} 946 \\
\left(107^{\prime} 299\right)\end{array}$ \\
\hline Net sales/revenue & $\begin{array}{l}2^{\prime} 080^{\prime} 307 \\
\left(5^{\prime} 999^{\prime} 574\right)\end{array}$ & $\begin{array}{l}4^{\prime} 008^{\prime} 797 \\
\left(6^{\prime} 327^{\prime} 785\right)\end{array}$ & $\begin{array}{l}903^{\prime} 188 \\
\left(1^{\prime} 931^{\prime} 426\right)\end{array}$ & $\begin{array}{l}1^{\prime} 303^{\prime} 391 \\
\left(4^{\prime} 742^{\prime} 154\right)\end{array}$ & $\begin{array}{l}5^{\prime} 068^{\prime} 722 \\
\left(10^{\prime} 822^{\prime} 010\right)\end{array}$ & $\begin{array}{l}2^{\prime} 698^{\prime} 204 \\
\left(4^{\prime} 348^{\prime} 997\right)\end{array}$ & $\begin{array}{l}2^{\prime} 014^{\prime} 019 \\
\left(2^{\prime} 710^{\prime} 452\right)\end{array}$ \\
\hline Operating income & $\begin{array}{l}210^{\prime} 896 \\
\left(770^{\prime} 553\right)\end{array}$ & $\begin{array}{l}790^{\prime} 968 \\
\left(1^{\prime} 333^{\prime} 413\right)\end{array}$ & $\begin{array}{l}45^{\prime} 420 \\
\left(75^{\prime} 525\right)\end{array}$ & $\begin{array}{l}148^{\prime} 610 \\
\left(794^{\prime} 780\right)\end{array}$ & $\begin{array}{l}368^{\prime} 497 \\
\left(787^{\prime} 769\right)\end{array}$ & $\begin{array}{l}593^{\prime} 360 \\
\left(1^{\prime} 533^{\prime} 302\right)\end{array}$ & $\begin{array}{l}99^{\prime} 351 \\
\left(174^{\prime} 578\right)\end{array}$ \\
\hline $\begin{array}{l}\text { Property, plant \& } \\
\text { equipment }\end{array}$ & $\begin{array}{l}1^{\prime} 809^{\prime} 203 \\
\left(5^{\prime} 777^{\prime} 290\right)\end{array}$ & $\begin{array}{l}8^{\prime} 757^{\prime} 473 \\
\left(15^{\prime} 597^{\prime} 523\right)\end{array}$ & $\begin{array}{l}386^{\prime} 647 \\
\left(696^{\prime} 792\right)\end{array}$ & $\begin{array}{l}1^{\prime} 180^{\prime} 317 \\
\left(3^{\prime} 544^{\prime} 595\right)\end{array}$ & $\begin{array}{l}2^{\prime} 867^{\prime} 700 \\
\left(4^{\prime} 477^{\prime} 604\right)\end{array}$ & $\begin{array}{l}4^{\prime} 358^{\prime} 604 \\
\left(10^{\prime} 930^{\prime} 187\right)\end{array}$ & $\begin{array}{l}174^{\prime} 127 \\
\left(275^{\prime} 023\right)\end{array}$ \\
\hline Total assets & $\begin{array}{l}2^{\prime} 949^{\prime} 463 \\
\left(7^{\prime} 949^{\prime} 340\right)\end{array}$ & $\begin{array}{l}10^{\prime} 413^{\prime} 775 \\
\left(17^{\prime} 595^{\prime} 469\right)\end{array}$ & $\begin{array}{l}820^{\prime} 756 \\
\left(1^{\prime} 518^{\prime} 989\right)\end{array}$ & $\begin{array}{l}2^{\prime} 075^{\prime} 357 \\
\left(5^{\prime} 691^{\prime} 152\right)\end{array}$ & $\begin{array}{l}5^{\prime} 530^{\prime} 127 \\
\left(9^{\prime} 364^{\prime} 225\right)\end{array}$ & $\begin{array}{l}6^{\prime} 335^{\prime} 425 \\
\left(14^{\prime} 766^{\prime} 495\right)\end{array}$ & $\begin{array}{l}986^{\prime} 179 \\
\left(1^{\prime} 426^{\prime} 701\right)\end{array}$ \\
\hline Total capita & $\begin{array}{l}2^{\prime} 006^{\prime} 143 \\
\left(5^{\prime} 513^{\prime} 969\right)\end{array}$ & $\begin{array}{l}7^{\prime} 222^{\prime} 339 \\
\left(12^{\prime} 818^{\prime} 106\right)\end{array}$ & $\begin{array}{l}522^{\prime} 274 \\
\left(933^{\prime} 753\right)\end{array}$ & $\begin{array}{l}1^{\prime} 620^{\prime} 807 \\
\left(4^{\prime} 349^{\prime} 689\right)\end{array}$ & $\begin{array}{l}3^{\prime} 127^{\prime} 830 \\
\left(4^{\prime} 708^{\prime} 630\right)\end{array}$ & $\begin{array}{l}5^{\prime} 259^{\prime} 803 \\
\left(12^{\prime} 343^{\prime} 758\right)\end{array}$ & $\begin{array}{l}582^{\prime} 645 \\
\left(870^{\prime} 767\right)\end{array}$ \\
\hline Total debt & $\begin{array}{l}1^{\prime} 064^{\prime} 594 \\
\left(3^{\prime} 259^{\prime} 394\right)\end{array}$ & $\begin{array}{l}4^{\prime} 279^{\prime} 212 \\
\left(8^{\prime} 894^{\prime} 715\right)\end{array}$ & $\begin{array}{l}222^{\prime} 679 \\
\left(428^{\prime} 368\right)\end{array}$ & $\begin{array}{l}777^{\prime} 017 \\
\left(1^{\prime} 886^{\prime} 739\right)\end{array}$ & $\begin{array}{l}1^{\prime} 914^{\prime} 840 \\
\left(3^{\prime} 188^{\prime} 635\right)\end{array}$ & $\begin{array}{l}2^{\prime} 265^{\prime} 442 \\
\left(5^{\prime} 233^{\prime} 062\right)\end{array}$ & $\begin{array}{l}103^{\prime} 329 \\
\left(180^{\prime} 688\right)\end{array}$ \\
\hline $\begin{array}{l}\text { Total share- } \\
\text { holder's equity }\end{array}$ & $\begin{array}{l}1^{\prime} 056^{\prime} 706 \\
\left(2^{\prime} 835^{\prime} 223\right)\end{array}$ & $\begin{array}{l}3^{\prime} 310^{\prime} 941 \\
\left(5^{\prime} 197^{\prime} 831\right)\end{array}$ & $\begin{array}{l}357^{\prime} 984 \\
\left(696^{\prime} 848\right)\end{array}$ & $\begin{array}{l}891^{\prime} 261 \\
\left(2^{\prime} 640^{\prime} 409\right)\end{array}$ & $\begin{array}{l}1^{\prime} 541^{\prime} 210 \\
\left(2^{\prime} 695^{\prime} 078\right)\end{array}$ & $\begin{array}{l}2^{\prime} 949^{\prime} 482 \\
\left(6^{\prime} 916^{\prime} 945\right)\end{array}$ & $\begin{array}{l}510^{\prime} 867 \\
\left(792^{\prime} 456\right)\end{array}$ \\
\hline
\end{tabular}


Table 10 Key financial figures (absolute) of logistics service providers used for regression analyses with beta as dependent variable

\begin{tabular}{|c|c|c|c|c|c|c|c|}
\hline Cluster description & & $\begin{array}{l}\text { Railroad } \\
\text { transportation }\end{array}$ & $\begin{array}{l}\text { Motor freight } \\
\text { transportation }\end{array}$ & $\begin{array}{l}\text { Water } \\
\text { transportation }\end{array}$ & $\begin{array}{l}\text { Transportation } \\
\text { by air }\end{array}$ & $\begin{array}{l}\text { Pipeline, except } \\
\text { natural gas }\end{array}$ & $\begin{array}{l}\text { Transportation } \\
\text { services }\end{array}$ \\
\hline Cluster (SIC code) & ALL LSPs & SIC 40 & SIC 42 & SIC 44 & SIC 45 & SIC 46 & SIC 47 \\
\hline Number of LSPs & 702 & 48 & 187 & 337 & 140 & 23 & 25 \\
\hline Absolute (US\$) & \multicolumn{7}{|c|}{ Mean value (standard deviation in parentheses) } \\
\hline $\begin{array}{l}\text { Cash flow per } \\
\text { share }\end{array}$ & $\begin{array}{l}9.21 \\
(107.45)\end{array}$ & $\begin{array}{l}6.54 \\
(18.71)\end{array}$ & $\begin{array}{l}1.46 \\
(57.74)\end{array}$ & $\begin{array}{l}12.11 \\
(138.86)\end{array}$ & $\begin{array}{l}8.24 \\
(67.11)\end{array}$ & $\begin{array}{l}48.33 \\
(199.66)\end{array}$ & $\begin{array}{l}0.75 \\
(1.18)\end{array}$ \\
\hline $\begin{array}{l}\text { Total current } \\
\text { assets }\end{array}$ & $\begin{array}{l}611^{\prime} 463 \\
\left(1^{\prime} 673^{\prime} 788\right)\end{array}$ & $\begin{array}{l}833^{\prime} 810 \\
\left(1^{\prime} 221^{\prime} 419\right)\end{array}$ & $\begin{array}{l}214^{\prime} 594 \\
\left(529^{\prime} 906\right)\end{array}$ & $\begin{array}{l}427^{\prime} 359 \\
\left(1^{\prime} 247^{\prime} 787\right)\end{array}$ & $\begin{array}{l}1^{\prime} 457^{\prime} 307 \\
\left(2^{\prime} 826^{\prime} 401\right)\end{array}$ & $\begin{array}{l}1^{\prime} 046^{\prime} 246 \\
\left(2^{\prime} 890^{\prime} 201\right)\end{array}$ & $\begin{array}{l}440^{\prime} 570 \\
\left(714^{\prime} 312\right)\end{array}$ \\
\hline $\begin{array}{l}\text { Total current } \\
\text { liabilities }\end{array}$ & $\begin{array}{l}561^{\prime} 216 \\
\left(1^{\prime} 643^{\prime} 365\right)\end{array}$ & $\begin{array}{l}1^{\prime} 123^{\prime} 661 \\
\left(2^{\prime} 246^{\prime} 278\right)\end{array}$ & $\begin{array}{l}179^{\prime} 729 \\
\left(441^{\prime} 518\right)\end{array}$ & $\begin{array}{l}308^{\prime} 133 \\
\left(947^{\prime} 317\right)\end{array}$ & $\begin{array}{l}1^{\prime} 524^{\prime} 440 \\
\left(2^{\prime} 967^{\prime} 761\right)\end{array}$ & $\begin{array}{l}568^{\prime} 284 \\
\left(1^{\prime} 150^{\prime} 455\right)\end{array}$ & $\begin{array}{l}294^{\prime} 013 \\
\left(496^{\prime} 098\right)\end{array}$ \\
\hline EBIT & $\begin{array}{l}158^{\prime} 989 \\
\left(663^{\prime} 820\right)\end{array}$ & $\begin{array}{l}620^{\prime} 750 \\
\left(1^{\prime} 189^{\prime} 785\right)\end{array}$ & $\begin{array}{l}35^{\prime} 612 \\
\left(75^{\prime} 501\right)\end{array}$ & $\begin{array}{l}106^{\prime} 245 \\
\left(620^{\prime} 160\right)\end{array}$ & $\begin{array}{l}265^{\prime} 244 \\
\left(765^{\prime} 348\right)\end{array}$ & $\begin{array}{l}379^{\prime} 730 \\
\left(1^{\prime} 221^{\prime} 440\right)\end{array}$ & $\begin{array}{l}76^{\prime} 518 \\
\left(160^{\prime} 361\right)\end{array}$ \\
\hline EBITDA & $\begin{array}{l}269^{\prime} 234 \\
\left(978^{\prime} 624\right)\end{array}$ & $\begin{array}{l}949^{\prime} 368 \\
\left(1^{\prime} 807^{\prime} 999\right)\end{array}$ & $\begin{array}{l}64^{\prime} 433 \\
\left(121^{\prime} 863\right)\end{array}$ & $\begin{array}{l}178^{\prime} 426 \\
\left(924^{\prime} 607\right)\end{array}$ & $\begin{array}{l}503^{\prime} 247 \\
\left(1^{\prime} 082^{\prime} 513\right)\end{array}$ & $\begin{array}{l}538^{\prime} 949 \\
\left(1^{\prime} 702^{\prime} 025\right)\end{array}$ & $\begin{array}{l}99^{\prime} 178 \\
\left(178^{\prime} 178\right)\end{array}$ \\
\hline Long term deb & $\begin{array}{l}816^{\prime} 868 \\
\left(2^{\prime} 557^{\prime} 209\right)\end{array}$ & $\begin{array}{l}2^{\prime} 901^{\prime} 161 \\
\left(6^{\prime} 860^{\prime} 048\right)\end{array}$ & $\begin{array}{l}159^{\prime} 940 \\
\left(454^{\prime} 107\right)\end{array}$ & $\begin{array}{l}586^{\prime} 662 \\
\left(1^{\prime} 537^{\prime} 707\right)\end{array}$ & $\begin{array}{l}1^{\prime} 502^{\prime} 034 \\
\left(2^{\prime} 741^{\prime} 534\right)\end{array}$ & $\begin{array}{l}1^{\prime} 730^{\prime} 225 \\
\left(3^{\prime} 943^{\prime} 672\right)\end{array}$ & $\begin{array}{l}42^{\prime} 301 \\
\left(78^{\prime} 686\right)\end{array}$ \\
\hline Net income & $\begin{array}{l}84^{\prime} 968 \\
\left(386^{\prime} 495\right)\end{array}$ & $\begin{array}{l}309^{\prime} 690 \\
\left(652^{\prime} 096\right)\end{array}$ & $\begin{array}{l}18^{\prime} 608 \\
\left(59^{\prime} 577\right)\end{array}$ & $\begin{array}{l}55^{\prime} 049 \\
\left(295^{\prime} 080\right)\end{array}$ & $\begin{array}{l}149^{\prime} 305 \\
\left(550^{\prime} 644\right)\end{array}$ & $\begin{array}{l}228^{\prime} 952 \\
\left(806^{\prime} 471\right)\end{array}$ & $\begin{array}{l}43^{\prime} 835 \\
\left(99^{\prime} 740\right)\end{array}$ \\
\hline Net sales/revenue & $\begin{array}{l}1^{\prime} 789^{\prime} 260 \\
\left(5^{\prime} 430^{\prime} 791\right)\end{array}$ & $\begin{array}{l}3^{\prime} 000^{\prime} 226 \\
\left(5^{\prime} 448^{\prime} 536\right)\end{array}$ & $\begin{array}{l}717^{\prime} 058 \\
\left(1^{\prime} 692^{\prime} 502\right)\end{array}$ & $\begin{array}{l}1^{\prime} 031^{\prime} 210 \\
\left(3^{\prime} 942^{\prime} 464\right)\end{array}$ & $\begin{array}{l}4^{\prime} 438^{\prime} 789 \\
\left(9^{\prime} 514^{\prime} 687\right)\end{array}$ & $\begin{array}{l}2^{\prime} 950^{\prime} 100 \\
\left(6^{\prime} 064^{\prime} 315\right)\end{array}$ & $\begin{array}{l}1^{\prime} 645^{\prime} 841 \\
\left(2^{\prime} 536^{\prime} 230\right)\end{array}$ \\
\hline Operating income & $\begin{array}{l}144^{\prime} 827 \\
\left(630^{\prime} 634\right)\end{array}$ & $\begin{array}{l}621^{\prime} 057 \\
\left(1^{\prime} 157^{\prime} 978\right)\end{array}$ & $\begin{array}{l}33^{\prime} 306 \\
\left(71^{\prime} 129\right)\end{array}$ & $\begin{array}{l}94^{\prime} 772 \\
\left(608^{\prime} 884\right)\end{array}$ & $\begin{array}{l}220^{\prime} 337 \\
\left(670^{\prime} 597\right)\end{array}$ & $\begin{array}{l}367^{\prime} 708 \\
\left(1^{\prime} 142^{\prime} 348\right)\end{array}$ & $\begin{array}{l}79^{\prime} 751 \\
\left(160^{\prime} 997\right)\end{array}$ \\
\hline $\begin{array}{l}\text { Property, plant \& } \\
\text { equipment }\end{array}$ & $\begin{array}{l}1^{\prime} 569^{\prime} 333 \\
\left(4^{\prime} 911^{\prime} 056\right)\end{array}$ & $\begin{array}{l}6^{\prime} 646^{\prime} 942 \\
\left(13^{\prime} 332^{\prime} 655\right)\end{array}$ & $\begin{array}{l}367^{\prime} 438 \\
\left(893^{\prime} 623\right)\end{array}$ & $\begin{array}{l}1^{\prime} 018^{\prime} 166 \\
\left(2^{\prime} 918^{\prime} 269\right)\end{array}$ & $\begin{array}{l}2^{\prime} 699^{\prime} 078 \\
\left(4^{\prime} 690^{\prime} 264\right)\end{array}$ & $\begin{array}{l}3^{\prime} 154^{\prime} 995 \\
\left(8^{\prime} 232^{\prime} 728\right)\end{array}$ & $\begin{array}{l}147^{\prime} 637 \\
\left(252^{\prime} 629\right)\end{array}$ \\
\hline Total assets & $\begin{array}{l}2^{\prime} 579^{\prime} 179 \\
\left(7^{\prime} 092^{\prime} 887\right)\end{array}$ & $\begin{array}{l}8^{\prime} 168^{\prime} 849 \\
\left(15^{\prime} 160^{\prime} 763\right)\end{array}$ & $\begin{array}{l}710^{\prime} 062 \\
\left(1^{\prime} 488^{\prime} 464\right)\end{array}$ & $\begin{array}{l}1^{\prime} 739^{\prime} 855 \\
\left(4^{\prime} 803^{\prime} 834\right)\end{array}$ & $\begin{array}{l}5^{\prime} 085^{\prime} 045 \\
\left(9^{\prime} 367^{\prime} 922\right)\end{array}$ & $\begin{array}{l}4^{\prime} 669^{\prime} 999 \\
\left(11^{\prime} 247^{\prime} 478\right)\end{array}$ & $\begin{array}{l}802^{\prime} 815 \\
\left(1^{\prime} 328^{\prime} 270\right)\end{array}$ \\
\hline Total capita & $\begin{array}{l}1^{\prime} 734^{\prime} 691 \\
\left(4^{\prime} 769^{\prime} 033\right)\end{array}$ & $\begin{array}{l}5^{\prime} 786^{\prime} 458 \\
\left(11^{\prime} 049^{\prime} 612\right)\end{array}$ & $\begin{array}{l}466^{\prime} 030 \\
\left(1^{\prime} 029^{\prime} 773\right)\end{array}$ & $\begin{array}{l}1^{\prime} 355^{\prime} 365 \\
\left(3^{\prime} 668^{\prime} 587\right)\end{array}$ & $\begin{array}{l}2^{\prime} 788^{\prime} 102 \\
\left(4^{\prime} 672^{\prime} 656\right)\end{array}$ & $\begin{array}{l}3^{\prime} 800^{\prime} 373 \\
\left(9^{\prime} 332^{\prime} 690\right)\end{array}$ & $\begin{array}{l}474^{\prime} 392 \\
\left(808^{\prime} 711\right)\end{array}$ \\
\hline Total debt & $\begin{array}{l}973^{\prime} 137 \\
\left(2^{\prime} 883^{\prime} 166\right)\end{array}$ & $\begin{array}{l}3^{\prime} 241^{\prime} 108 \\
\left(7^{\prime} 462^{\prime} 934\right)\end{array}$ & $\begin{array}{l}215^{\prime} 592 \\
\left(527^{\prime} 176\right)\end{array}$ & $\begin{array}{l}705^{\prime} 274 \\
\left(1^{\prime} 765^{\prime} 481\right)\end{array}$ & $\begin{array}{l}1^{\prime} 848^{\prime} 680 \\
\left(3^{\prime} 352^{\prime} 426\right)\end{array}$ & $\begin{array}{l}1^{\prime} 830^{\prime} 115 \\
\left(4^{\prime} 062^{\prime} 999\right)\end{array}$ & $\begin{array}{l}87^{\prime} 670 \\
\left(165^{\prime} 016\right)\end{array}$ \\
\hline $\begin{array}{l}\text { Total share- } \\
\text { holder's equity }\end{array}$ & $\begin{array}{l}872^{\prime} 530 \\
\left(2^{\prime} 466^{\prime} 281\right)\end{array}$ & $\begin{array}{l}2^{\prime} 840^{\prime} 442 \\
\left(4^{\prime} 861^{\prime} 755\right)\end{array}$ & $\begin{array}{l}298^{\prime} 170 \\
\left(659^{\prime} 711\right)\end{array}$ & $\begin{array}{l}706^{\prime} 458 \\
\left(2^{\prime} 123^{\prime} 467\right)\end{array}$ & $\begin{array}{l}1^{\prime} 238^{\prime} 929 \\
\left(2^{\prime} 542^{\prime} 922\right)\end{array}$ & $\begin{array}{l}1^{\prime} 984^{\prime} 269 \\
\left(5^{\prime} 183^{\prime} 103\right)\end{array}$ & $\begin{array}{l}413^{\prime} 981 \\
\left(734^{\prime} 967\right)\end{array}$ \\
\hline
\end{tabular}


Table 11 Characterization of microeconomic variables and related studies on CoC of LSPs

\begin{tabular}{|c|c|c|c|}
\hline $\begin{array}{l}\text { Microeconomic } \\
\text { variable }\end{array}$ & Definition & Importance and relevance for LSPs & Studies \\
\hline \multicolumn{4}{|l|}{ Asset structure } \\
\hline $\begin{array}{l}\text { Intensity of } \\
\text { investment }\end{array}$ & $\begin{array}{l}\text { Non-current assets/ } \\
\text { total assets }\end{array}$ & \multirow{6}{*}{$\begin{array}{l}\text { Microeconomic variables related to the asset structure of an LSP } \\
\text { allow for conclusions on their scope of services (LSPs taking the } \\
\text { role of coordination or provision of asset-based services), the } \\
\text { degree of customization, and average duration of business } \\
\text { relationships, as well as on LSPs' asset flexibility [21] }\end{array}$} & $\begin{array}{l}\text { Based on the analyzed } \\
\text { variables of asset structure } \\
{[37,40,47,68]}\end{array}$ \\
\hline Asset intensity 1 & $\begin{array}{l}\text { Non-current assets/ } \\
\text { current assets }\end{array}$ & & {$[40,47]$} \\
\hline $\begin{array}{l}\text { Continuous } \\
\text { intensity }\end{array}$ & $\begin{array}{l}\text { Current assets/total } \\
\text { assets }\end{array}$ & & $\begin{array}{l}\text { Based on the analyzed } \\
\text { variables of asset structure } \\
{[37,40,47,68]}\end{array}$ \\
\hline Asset intensity 2 & $\begin{array}{l}\text { Current assets/non- } \\
\text { current assets }\end{array}$ & & {$[40,47]$} \\
\hline Asset turnover & $\begin{array}{l}\text { Annual revenues/total } \\
\text { assets }\end{array}$ & & {$[37,68]$} \\
\hline $\begin{array}{l}\text { Current asset } \\
\text { turnover }\end{array}$ & $\begin{array}{l}\text { Annual revenues/ } \\
\text { current assets }\end{array}$ & & $\begin{array}{l}\text { Based on the analyzed } \\
\text { variables of asset structure } \\
{[37,40,47,68]}\end{array}$ \\
\hline \multicolumn{4}{|l|}{ Capital structure } \\
\hline $\begin{array}{l}\text { Debt to equity } \\
\text { ratio }\end{array}$ & Debt/equity & \multirow{3}{*}{$\begin{array}{l}\text { Microeconomic variables related to the capital structure of an LSP } \\
\text { allow for conclusions on how assets are financed, by debt or } \\
\text { equity [78] }\end{array}$} & {$[47,79]$} \\
\hline Equity ratio & Equity/total capital & & {$[16,40]$} \\
\hline Debt ratio & Debt/total capital & & {$[37,80]$} \\
\hline \multicolumn{4}{|l|}{ Liquidity structure } \\
\hline Quick ratio & $\begin{array}{l}\text { (Current assets- } \\
\text { inventories)/current } \\
\text { liabilities }\end{array}$ & $\begin{array}{l}\text { Microeconomic variables related to the liquidity structure of an } \\
\text { LSP reflects the ability of LSPs to pay all outstanding claims and } \\
\text { cover liabilities [21] }\end{array}$ & {$[37,68]$} \\
\hline Current ratio & $\begin{array}{l}\text { Current assets/current } \\
\text { liabilities }\end{array}$ & & {$[79,81]$} \\
\hline \multicolumn{4}{|c|}{ Profitability structure } \\
\hline $\begin{array}{l}\text { Return on } \\
\text { equity (ROE) }\end{array}$ & $\begin{array}{l}\text { Net income/ } \\
\text { shareholders' equity }\end{array}$ & \multirow[t]{3}{*}{$\begin{array}{l}\text { Microeconomic variables related to the profitability structure of an } \\
\text { LSP allow for conclusions on the financial performance of LSPs }\end{array}$} & {$[40],[67]$} \\
\hline $\begin{array}{l}\text { Return on assets } \\
\text { (ROA) }\end{array}$ & $\begin{array}{l}\text { Net income/total } \\
\text { assets }\end{array}$ & & {$[37,40,68,81]$} \\
\hline $\begin{array}{l}\text { Net profit } \\
\text { margin }\end{array}$ & Net income/revenues & & {$[40,81]$} \\
\hline
\end{tabular}




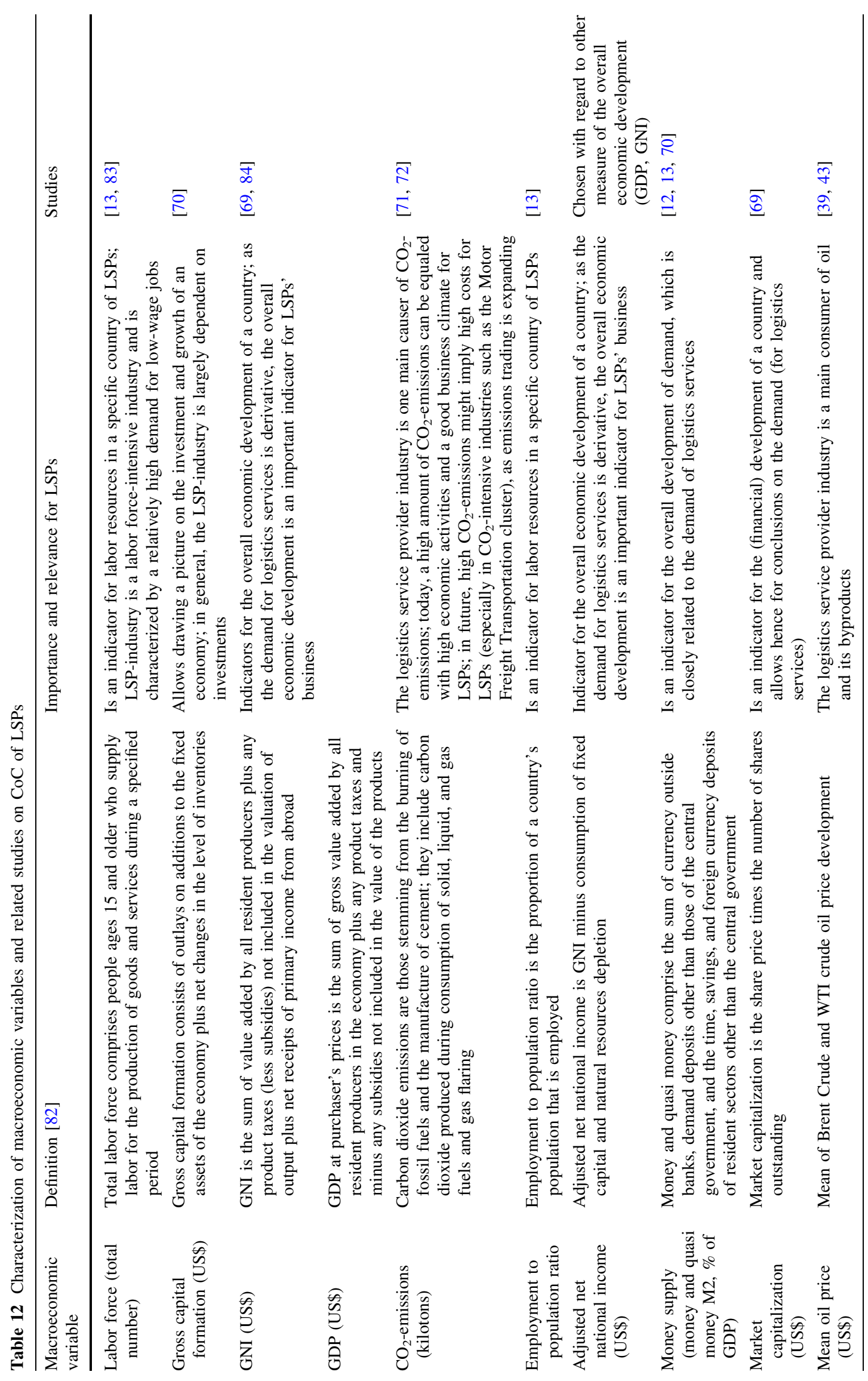




\section{References}

1. Bowersox DJ, Closs DJ, Cooper MB (2007) Supply chain logistics management. McGraw-Hill Higher Education, Maidenhead

2. Fugate BS, Mentzer JT, Flint DJ (2008) The role of logistics in market orientation. J Bus Logist 29:1-26

3. Grant RM (1991) The resource-based theory of competitive advantage: implications for strategy formulation. Calif Manag Rev 33:114-135

4. Apergis N, Artikis G, Eleftheriou S, Sorros J (2011) Accounting information and cost of capital: a theoretical approach. Modern Econ 2:589-596

5. Ogier T, Rugman J, Spicer L (2012) The real cost of capital. Pearson Education Limited, Upper Saddle River

6. Easley D, O'hara M (2004) Information and the cost of capital. J Financ 59:1553-1583

7. Häkkinen L, Norrman A, Hilmola O-P, Ojala L (2004) Logistics integration in horizontal mergers and acquisitions. Int $\mathrm{J}$ Logisti Manag 15:27-42

8. Daugherty PJ, Chen H, Ferrin BG (2011) Organizational structure and logistics service innovation. Int J Logisti Manag 22:26-51

9. Elyasiani E, Mansur I, Odusami B (2011) Oil price shocks and industry stock returns. Energy Econ 33:966-974

10. Driesprong G, Jacobsen B, Maat B (2008) striking oil: another puzzle? J Financ Econ 89:307-327

11. Huang X, Gattiker TF, Schwarz JL (2008) Interpersonal trust formation during the supplier selection process: the role of the communication channel. J Supply Chain Manag 44:53-75

12. Abugri BA (2006) Empirical relationship between macroeconomic volatility and stock returns: evidence from latin American markets. Int Rev Financ Anal 17:396-410

13. Rapach DE, Wohar ME, Rangvid J (2005) Macro variables and international stock return predictability. Int $\mathrm{J}$ Forecast 21:137-166

14. Cavaglia S, Brightman C, Aked M (2000) The increasing importance of industry factors. Financ Anal J 56:41-54

15. Sadorsky P (1999) Oil price shocks and stock market activity. Energy Econ 21:449-469

16. Kavussanos MG, Marcoulis SN (1997) The stock market perception of industry risk and microeconomic factors: the case of the US water transportation industry versus other transport industries. Transport Res Part E Logist Transport Rev 33:147-158

17. Kaneko T, Lee B-S (1995) Relative importance of economic factors in the US and Japanese stock markets. J Jpn Int Econ 9:290-307

18. Fama EF, French KR (1992) The cross-section of expected stock returns. J Financ 47:427-465

19. Ross SA (1976) The arbitrage theory of capital asset pricing. J Econ Theory 13:341-360

20. King BF (1966) Market and industry factors in stock price behavior. J Bus 39:139-190

21. Hofmann E, Lampe K (2013) Financial statement analysis of logistics service providers: ways of enhancing performance. Int $\mathbf{J}$ Phys Distrib Logist Manag 43:321-342

22. Liu C-L, Lyons AC (2011) An analysis of third-party logistics performance and service provision. Transport Res Part E Logist Transport Rev 47:547-570

23. Töyli J, Häkkinen L, Ojala L, Naula T (2008) Logistics and financial performance: an analysis of 424 Finnish small and medium-sized enterprises. Int J Phys Distrib Logist Manag 38:57-80

24. Panayides PM (2007) The impact of organizational learning on relationship orientation, logistics service effectiveness and performance. Ind Mark Manage 36:68-80
25. Panayides PM, So M (2005) The impact of integrated logistics relationships on third-party logistics service quality and performance. Maritime Econ Logist 7:36-55

26. Ellinger AE, Lynch DF, Hansen JD (2003) Firm size, web site content, and financial performance in the transportation industry. Ind Mark Manage 32:177-185

27. Drobetz W, Gounopoulos D, Merikas A, Schröder H (2013) Capital structure decisions of globally-listed shipping companies. Transport Res Part E Logist Transport Rev 52:49-76

28. Besley S, Brigham EF (2008) Essentials of managerial finance. Thomson/South-Western, Mason

29. Copeland T, Koller T, Murrin J (2000) Valuation. Wiley, New York

30. Baker HK, Powell G (2009) Understanding financial management: a practical guide. Wiley, Hoboken

31. Armitage S (2005) The cost of capital: intermediate theory. Cambridge University Press, Cambridge

32. Pratt SP (2003) Cost of capital: estimation and applications. Wiley, Hoboken

33. Isakov D, Sonney F (2002) Are practitioners right? On the relative importance of industrial factors in international stock returns. EFMA 2002 London Meetings

34. Baca SP, Garbe BL, Weiss RA (2000) The rise of sector effects in major equity markets. Financ Anal J 56:34-40

35. Huang R, Masulis R, Stoll H (1996) Energy shocks and financial markets. J Futur Markets 16:1-27

36. Levy RA (1974) Beta coefficients as predictors of return. Financ Anal J 30:61-69

37. Iqbal MJ, Ali Shah SZ (2012) Determinants of systematic risk. J Commer 4:47-56

38. Hong G, Sarkar S (2007) Equity systematic risk (beta) and its determinants. Contemp Account Res 24:423-466

39. Arfaoui M, Abaoub E (2010) The determinants of systematic risk: international evidence from the macro-finance interface. J Adv Stud Financ 1:121-143

40. Martikainen $T$ (1991) On the significance of the economic determinants of systematic risk: empirical evidence with finnish data. Appl Financ Econ 1:97-104

41. Houmes RE, MacArthur JB, Stranahan H (2012) The operating leverage impact on systematic risk within a context of choice: an analysis of the US trucking industry. Manag Financ 38:1184-1202

42. MacArthur JB, Houmes RE, Stranahan HA (2008) A study of the relationship between strategic cost structure choice and stock return behavior in the transportation industry: preliminary results. ASBBS E-J 4:122-127

43. Lu J-R, Chen C-C (2010) Effect of oil price risk on systematic risk from transportation services industry evidence. Serv Ind J 30:1853-1870

44. Kavussanos MG, Marcoulis SN (1998) Beta comparisons across industries-a water transportation industry perspective. Maritime Policy Manag 25:175-184

45. Allen SA, Cunningham LF, Wood WR (1990) Airline industry deregulation and changes in systematic risk: introduction background industry betas hypothesis fundamental characteristics of industry deregulation's effect of airline characteristics earnings covariability and pricing freedoms operating. Transportation (1986-1998) 17:49-66

46. Bancel F, Mittoo UR (2004) Cross-country determinants of capital structure choice: a survey of European Firms. Financ Manage 33:103-132

47. Sudarsanam PS (1992) Market and industry structure and corporate cost of capital. J Indus Econ 40:189-199

48. Brooks R, Del Negro M (2005) A latent factor model with global, country, and industry shocks for international stock returns. IMF working paper 
49. Gebhardt WR, Lee CMC, Swaminathan B (2001) Toward an implied cost of capital. J Account Res 39:135-176

50. El Ghoul S, Guedhami O, Kwok CCY, Mishra DR (2011) Does corporate social responsibility affect the cost of capital? J Bank Financ 35:2388-2406

51. Rajan RG, Zingales L (1996) Financial dependence and growth. National Bureau of Economic Research, Cambridge

52. Fama EF, French KR (1997) Industry costs of equity. J Financ Econ 43:153-193

53. Titman S, Wessels R (1988) The determinants of capital structure choice. J Financ 43:1-19

54. Balakrishnan S, Fox I (1993) Asset specificity, firm heterogeneity and capital structure. Strateg Manag J 14:3-16

55. Fama EF, MacBeth JD (1973) Risk, return, and equilibrium: empirical tests. J Political Econ 81:607-636

56. Chen N-F, Roll R, Ross SA (1986) Economic forces and the stock market. J Bus 59:383-403

57. Sousa R, Voss CA (2008) Contingency research in operations management practices. J Oper Manag 26:697-713

58. Chow G, Heaver TD, Henriksson LE (1995) Strategy, structure and performance: a framework for logistics research. Logist Transport Rev 31:285

59. Weiers RM, Gray JB, Peters LH (2008) Intro to business statistics. South-Western Cenagee Learning, Independence

60. US Department of Labor (2013) SIC division structure. https:// www.osha.gov/pls/imis/sic_manual.html. Accessed 5 Mar 2013

61. The World Bank (2013) Country and lending groups. http://data. worldbank.org/about/country-classifications/country-and-lendinggroups. Accessed 5 Mar 2013

62. Statista (2013) Statistics and market data on minerals, oil \& refinery. http://www.statista.com/markets/7/topic/57/mineralsoil-refinery/. Accessed 12 Mar 2013

63. Damodaran A (2014) The data page. http://pages.stern.nyu.edu/ adamodar/. Accessed 26 Jun 2014

64. Ross SA, Westerfield RW, Jaffe JF (2008) Corporate finance. McGraw-Hill Higher Education, Maidenhead

65. Modigliani F, Miller M (1958) The cost of capital, corporation finance and the theory of investment. Am Econ Rev 48:261-297

66. Horngren CT, Harrison WT, Oliver MS (2012) Financial and managerial accounting. Prentice Hall, Upper Saddle River

67. Jung H-I (2008) WACC as the touchstone performance indicator. Int J Contemp Hosp Manag 20:700-710
68. Lee J-S, Jang SS (2007) The systematic-risk determinants of the US Airline industry. Tour Manag 28:434-442

69. Patro DK, Wald JK, Wu Y (2002) The impact of macroeconomic and financial variables on market risk: evidence from international equity returns. Eur Financ Manag 8:421-447

70. Mohammad SD, Hussain A, Ali A (2009) Impact of macroeconomics variables on stock prices: empirical evidence in case of KSE (Karachi Stock Exchange). Eur J Sci Res 38:96-103

71. Busch T (2006) Emissions Trading and Effects on Financial Markets. In: Antes R, Hansjürgens B, Letmathe P (eds) Emissions trading and business. Physica, Heidelberg, pp 257-272

72. Yamaguchi K (2008) Reexamination of stock price reaction to environmental performance: a GARCH application. Ecol Econ 68:345-352

73. Copeland TE, Weston JF, Shastri K (2005) Financial theory and corporate policy: students solutions manual. Addison Wesley Publishing, Brigham

74. Fama EF, French KR (1993) Common risk factors in the returns on stocks and bonds. J Financ Econ 33:3-56

75. Koller T, Goedhart M, Wessels D (2010) Valuation: measuring and managing the value of companies. Wiley, Hoboken

76. Pratt SP, Grabowski RJ (2010) Cost of capital: applications and examples. Wiley, Hoboken

77. DeFusco RA, McLeavey DW, Pinto JE et al (2011) Quantitative investment analysis. Wiley, Hoboken

78. Modigliani F, Miller MH (1958) The cost of capital, corporation finance and the theory of investment. Am Econ Rev 48:261-297

79. Moyer RC, Chatfield R (1983) Market power and systematic risk. J Econ Bus 35:123-130

80. Apergis N, Artikis G, Eleftheriou S, Sorros J (2012) Accounting information, the cost of capital and excess stock returns: the role of earnings quality-evidence from panel data. Int Bus Res 5:123-136

81. Logue DE, Merville LJ (1972) Financial policy and market expectations. Finance Manag 1:37

82. The World Bank (2014) Indicators. http://data.worldbank.org/ indicator. Accessed 14 Jun 2014

83. Boyd JH, Hu J, Jagannathan R (2005) The stock market's reaction to unemployment news: why bad news is usually good for stocks. J Financ 60:649-672

84. Ferson WE, Harvey CR (1997) Fundamental determinants of national equity market returns: a perspective on conditional asset pricing. J Bank Financ 21:1625-1665 